ESAIM: M2AN 48 (2014) 1431-1449

DOI: $10.1051 / \mathrm{m} 2 \mathrm{an} / 2014002$
ESAIM: Mathematical Modelling and Numerical Analysis

www.esaim-m2an.org

\title{
EQUIVALENT BOUNDARY CONDITIONS FOR AN ELASTO-ACOUSTIC PROBLEM SET IN A DOMAIN WITH A THIN LAYER*
}

\author{
VICTOR PÉRON ${ }^{1}$
}

\begin{abstract}
We present equivalent conditions and asymptotic models for the diffraction problem of elastic and acoustic waves in a solid medium surrounded by a thin layer of fluid medium. Due to the thinness of the layer with respect to the wavelength, this problem is well suited for the notion of equivalent conditions and the effect of the fluid medium on the solid is as a first approximation local. We derive and validate equivalent conditions up to the fourth order for the elastic displacement. These conditions approximate the acoustic waves which propagate in the fluid region. This approach leads to solve only elastic equations. The construction of equivalent conditions is based on a multiscale expansion in power series of the thickness of the layer for the solution of the transmission problem.
\end{abstract}

Mathematics Subject Classification. 35C20, 35J25, 41A60, 74F10.

Received July 10, 2013. Revised December 13, 2013.

Published online August 13, 2014.

\section{INTRODUCTION}

The concept of Equivalent Boundary Conditions (also called approximate, effective, or impedance conditions) is classical in the modeling of wave propagation phenomena. Equivalent Conditions (ECs) are usually introduced to reduce the computational domain. The main idea consists to replace an "exact" model inside a part of the domain (for instance a thin layer of dielectric or a highly absorbing material) by an approximate boundary condition. This idea is pertinent when the effective condition can be readily handled for numerical computations, for instance when this condition is local [6,12,13,25]. In the 1990's Engquist-Nédélec [12], Abboud-Ammari [1], Bendali-Lemrabet [6], Ammari-Nédélec [3], and Lafitte [16] derived equivalent conditions for acoustic and electromagnetic scattering problems to approximate an obstacle coated by a thin layer of dielectric (absorbing) material inside the domain of interest. Impedance conditions are also used to reduce the computational domain for scattering problems from highly absorbing obstacles [5, 13, 17, 19, 24, 25].

The main application of this work concern the mathematical modeling of earthquake on the Earth's surface. The simulation of large-scale geophysics phenomena represents a main challenge for our society. Seismic activities worldwide have shown how crucial it is to enhance our understanding of the impact of earthquakes. In this context, the coupling of elastic and acoustic waves equations is essential if we want to reproduce real physical

Keywords and phrases. Asymptotic expansions, equivalent boundary conditions, elasto-acoustic coupling.

* This work was supported by the project HPC GA 295217 - IRSES2011.

1 LMAP CNRS UMR 5142 \& Team MAGIQUE 3D INRIA Bordeaux Sud-Ouest, Université de Pau et des Pays de l'Adour, Avenue de l'Université, BP 1155, 64013 Pau Cedex, France. victor.peron@univ-pau.fr 
phenomena such as an earthquake. We can thus take into account the effects of the ocean on the propagation of seismic waves. Elasto-acoustic coupling problems are rather classical in the mathematical modeling of wave propagation phenomena. We refer to several works in references $[2,11,14,15,20-22]$ and the monography [9], Section 5.4.e which concern the direct problem for fluid-structure interaction systems and acoustic (or elastic) scattering by smooth elastic obstacles. We intend to work in the context of this application for which we consider that the medium consists of land areas surrounded by fluid zones whose thickness is very small, typically with respect to the wavelength. This raises the difficulty of applying a finite element method on a mesh that combines fine cells in the fluid zone and much larger cells in the solid zone. To overcome this difficulty and to solve this problem, we adopt an asymptotic method which consists to "approximate" the fluid portion by an equivalent boundary condition. This boundary condition is then coupled with the elastic wave equation and a finite element method can be applied to solve the resulting boundary value problem.

In this paper, we present elements of derivation together with mathematical justifications for equivalent boundary conditions, which appear as a first, second, third or fourth order approximations (Sect. 2.4) with respect to the small parameter (the thickness of the fluid layer) and satisfied by the elastic displacement $\mathbf{u}$. This work is concerned essentially with theoretical objectives. The numerical pertinence of these ECs up to the third order have already been shown for the two-dimensional problem [10].

There are several similarities in this paper and in the works in references $[1,6,12]$ in which the authors considered the problem of a time-harmonic wave for the Helmholtz equation. In reference [12], the authors construct ECs up to order 3 whereas in Reference [6] the authors derive and analyze ECs up to order 4. In this paper, we construct and analyze ECs up to order 4 for elasto-acoustics. As in references [6,12], the construction of the ECs relies on a multiscale expansion of the exact solution in power series of the small parameter. However, there are several differences between the results of this paper and the works in references $[6,12]$ since ECs are not of the same nature. The ECs are of "u $\cdot \mathbf{n}$-to-T(u)" nature for elasto-acoustics since a local impedance operator links the normal traces of $\mathbf{u}$ and the stress vector $\mathbf{T}(\mathbf{u})$ whereas the ECs are of "Dirichlet-to-Neumann" nature for acoustics in references [6,12]. One difficulty to validate the equivalent conditions lies in the proof of uniform energy estimates for the exact solution of the elasto-acoustic coupling. We overcome this difficulty using a compactness argument and removing a discret set of resonant frequencies (which may appear in the solid part of the domain). We revisit the proof of uniform estimates in Reference [6]. We can not apply straightforwardly the proof of estimates in Reference [6] to the elasto-acoustic coupling since the transmission conditions (which are purely natural) for elasto-acoustics appear in the weak formulation and play a crucial role. We prove wellposedness and convergence results for ECs up to the fourth order.

The outline of the paper proceeds as follows. In Section 2, we introduce the mathematical model and the framework for the elasto-acoustic problem and equivalent conditions. We present briefly a formal derivation of equivalent conditions. Then we present uniform estimates for the solution of the transmission problem. In Section 3, we present equivalent conditions and asymptotic models associated with the solution of the exact problem. In Section 4, we prove uniform estimates for the solution of the elasto-acoustic problem. In Section 5, we derive and validate a two-scale asymptotic expansion at any order for the solution of the problem, and we construct formally ECs. In Section 6, we prove stability results for ECs and the convergence of ECs towards the exact model.

\section{The MATHEMATICAL MODEL}

In this section, we introduce the model problem (Sect. 2.2) and the framework for the elasto-acoustic problem. Then we remind the definition of equivalent conditions and we state uniform estimates for the solution of the exact problem. We start this section with a formal derivation of the approximate boundary conditions.

\subsection{Formal derivation of equivalent conditions}

In this section, we present briefly a formal derivation of equivalent conditions. We summarize this process in two steps. All the details and formal calculi are presented in Section 5. 
First step: A multiscale expansion

The first step consists to derive a multiscale expansion for the solution $\left(\mathbf{u}_{\varepsilon}, \mathbf{p}_{\varepsilon}\right)$ of the model problem $(2.1)$ (Sect. 2.2): it possesses an asymptotic expansion in power series of the small parameter $\varepsilon$

$$
\begin{gathered}
\mathbf{u}_{\varepsilon}(\mathbf{x})=\mathbf{u}_{0}(\mathbf{x})+\varepsilon \mathbf{u}_{1}(\mathbf{x})+\varepsilon^{2} \mathbf{u}_{2}(\mathbf{x})+\ldots \quad \text { in } \Omega_{\mathbf{s}}, \\
\mathbf{p}_{\varepsilon}(\mathbf{x})=\mathrm{p}_{0}(\mathbf{x} ; \varepsilon)+\varepsilon \mathbf{p}_{1}(\mathbf{x} ; \varepsilon)+\varepsilon^{2} \mathbf{p}_{2}(\mathbf{x} ; \varepsilon)+\ldots \text { in } \Omega_{\mathrm{f}}^{\varepsilon}, \\
\text { with } \mathbf{p}_{j}(\mathbf{x} ; \varepsilon)=\mathfrak{p}_{j}\left(y_{\alpha}, \frac{y_{3}}{\varepsilon}\right) .
\end{gathered}
$$

Here $\mathbf{x} \in \mathbb{R}^{3}$ are the cartesian coordinates and $\left(y_{\alpha}, y_{3}\right)$ is a "normal coordinate system" [8,23] to the surface $\Gamma=\partial \Omega_{\mathrm{s}}$ on the manifold $\Omega_{\mathrm{f}}^{\varepsilon}: y_{\alpha}(\alpha \in\{1,2\})$ is a tangential coordinate on $\Gamma$ and $y_{3} \in(0, \varepsilon)$ is the distance to the surface $\Gamma$. The term $\mathfrak{p}_{j}$ is a "profile" defined on $\Gamma \times(0,1)$. Formal calculi are presented in Section 5.1 and the first terms $\left(\mathfrak{p}_{j}, \mathbf{u}_{j}\right)$ for $j=0,1,2,3$ are explicited in Section 5.2.

\section{Second step: Construction of equivalent conditions}

The second step consists to identify for all $k \in\{0,1,2,3\}$ a simpler problem satisfied by the truncated expansion

$$
\mathbf{u}_{k, \varepsilon}:=\mathbf{u}_{0}+\varepsilon \mathbf{u}_{1}+\varepsilon^{2} \mathbf{u}_{2}+\ldots+\varepsilon^{k} \mathbf{u}_{k}
$$

up to a residual term in $\mathcal{O}\left(\varepsilon^{k+1}\right)$. The simpler problem writes

$$
\begin{cases}\nabla \cdot \underline{\underline{\sigma}}\left(\mathbf{u}_{\varepsilon}^{k}\right)+\omega^{2} \rho \mathbf{u}_{\varepsilon}^{k}=\mathbf{f} & \text { in } \quad \Omega_{\mathbf{s}} \\ \mathbf{T}\left(\mathbf{u}_{\varepsilon}^{k}\right)+\mathrm{B}_{k, \varepsilon}\left(\mathbf{u}_{\varepsilon}^{k} \cdot \mathbf{n}\right) \mathbf{n}=0 & \text { on } \quad \Gamma .\end{cases}
$$

Here $\mathbf{f}$ is the data of the model problem (2.1) and $B_{k, \varepsilon}$ is a surfacic differential operator acting on functions defined on $\Gamma$ and which depends on $\varepsilon$

$$
\begin{gathered}
\mathrm{B}_{0, \varepsilon}=0 \\
\mathrm{~B}_{1, \varepsilon}(\mathrm{u})=-\varepsilon \omega^{2} \rho_{\mathrm{f}} \mathrm{u}, \\
\mathrm{B}_{2, \varepsilon}(\mathbf{u})=-\varepsilon \omega^{2} \rho_{\mathrm{f}}\left(1-\varepsilon \mathcal{H}\left(y_{\alpha}\right)\right) \mathrm{u} \\
\mathrm{B}_{3, \varepsilon}(\mathbf{u})=-\varepsilon \omega^{2} \rho_{\mathrm{f}}\left(1-\varepsilon \mathcal{H}\left(y_{\alpha}\right)+\frac{\varepsilon^{2}}{3}\left[\Delta_{\Gamma}+\kappa^{2} \mathbb{I}+4 \mathcal{H}^{2}\left(y_{\alpha}\right)-\mathcal{K}\left(y_{\alpha}\right)\right]\right) \mathbf{u} .
\end{gathered}
$$

Here $\mathcal{H}$ and $\mathcal{K}$ denote the mean curvature and the Gaussian curvature of the surface $\Gamma$ and $\Delta_{\Gamma}$ is the Laplace-Beltrami operator along $\Gamma$. Equivalent conditions are stated in Section 3.1. The construction of these conditions is detailed in Section 5.3.

\subsection{The model problem}

Our interest lies in an elasto-acoustic wave propagation problem in time-harmonic regime set in a domain with a thin layer. We consider the fluid-solid transmission problem

$$
\begin{cases}\Delta \mathbf{p}_{\varepsilon}+\kappa^{2} \mathbf{p}_{\varepsilon}=0 & \text { in } \Omega_{\mathrm{f}}^{\varepsilon} \\ \nabla \cdot \underline{\underline{\sigma}}\left(\mathbf{u}_{\varepsilon}\right)+\omega^{2} \rho \mathbf{u}_{\varepsilon}=\mathbf{f} & \text { in } \Omega_{\mathrm{s}} \\ \partial_{\mathbf{n}} \mathbf{p}_{\varepsilon}=\rho_{\mathrm{f}} \omega^{2} \mathbf{u}_{\varepsilon} \cdot \mathbf{n} & \text { on } \Gamma \\ \mathbf{T}\left(\mathbf{u}_{\varepsilon}\right)=-\mathbf{p}_{\varepsilon} \mathbf{n} & \text { on } \Gamma \\ \mathbf{p}_{\varepsilon}=0 & \text { on } \Gamma^{\varepsilon}\end{cases}
$$

set in a smooth bounded simply connected domain $\Omega^{\varepsilon}$ in $\mathbb{R}^{3}$ made of a solid, elastic object occupying a smooth connected subdomain $\Omega_{\mathrm{s}}$ entirely immersed in a fluid region occupying the subdomain $\Omega_{\mathrm{f}}^{\varepsilon}$. The domain $\Omega_{\mathrm{f}}^{\varepsilon}$ is a 


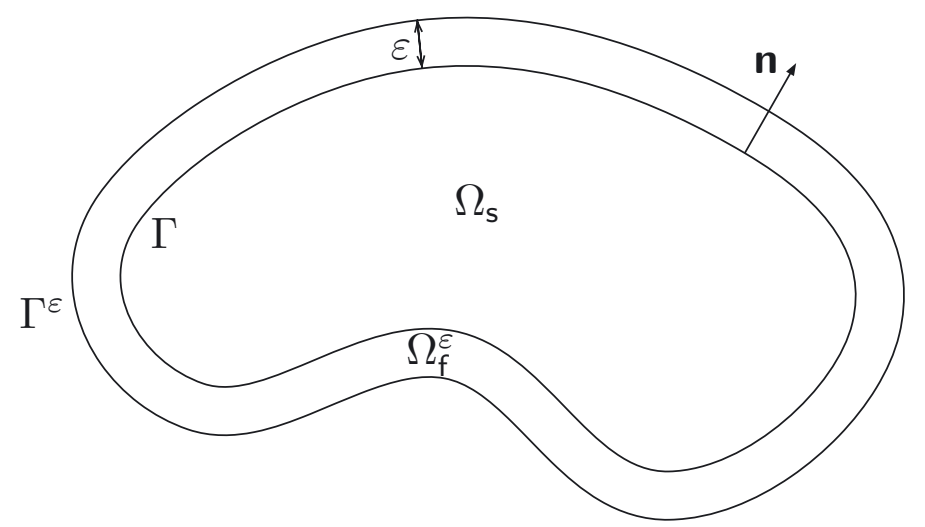

Figure 1. A cross-section of the domain $\Omega^{\varepsilon}$ and its subdomains $\Omega_{\mathrm{s}}$ and $\Omega_{\mathrm{f}}^{\varepsilon}$.

thin layer of uniform thickness $\varepsilon$ (i.e. the euclidean distance between surfaces $\Gamma$ and $\Gamma^{\varepsilon}$ is $\varepsilon$ ), see Figure 1 . We denote by $\Gamma^{\varepsilon}$ the boundary of the domain $\Omega^{\varepsilon}$, and by $\Gamma$ the interface between the subdomains $\Omega_{\mathrm{f}}^{\varepsilon}$ and $\Omega_{\mathrm{s}}$. We denote by $\mathbf{n}$ the unit normal to $\Gamma$ oriented from $\Omega_{\mathrm{s}}$ to $\Omega_{\mathrm{f}}^{\varepsilon}$.

In the elasto-acoustic system (2.1), we denote the unknowns by $\mathbf{u}_{\varepsilon}$ for the elastic displacement and by $\mathrm{p}_{\varepsilon}$ for the acoustic pressure. The time-harmonic wave field with angular frequency $\omega$ is characterized by using the Helmholtz equation for the pressure $\mathrm{p}_{\varepsilon}$, and by using an anisotropic discontinuous linear elasticity system for the displacement $\mathbf{u}_{\varepsilon}$. These equations contain several physical constants: $\kappa=\omega / c$ is the acoustic wave number, $c$ is the speed of the sound, $\rho$ is the density of the solid, and $\rho_{\mathrm{f}}$ is the density of the fluid. All these constants are independent of $\varepsilon$.

In the linear elastic equation, $\nabla \cdot$ denotes the divergence operator for tensors, $\underline{\underline{\sigma}}(\mathbf{u})$ is the stress tensor given by Hooke's law

$$
\underline{\underline{\sigma}}(\mathbf{u})=\underline{\underline{C}} \underline{\underline{\epsilon}}(\mathbf{u}) .
$$

Here $\underline{\underline{\epsilon}}(\mathbf{u})=\frac{1}{2}\left(\underline{\underline{\nabla}} \mathbf{u}+\underline{\underline{\nabla}} \mathbf{u}^{T}\right)$ is the strain tensor where $\underline{\underline{\nabla}}$ denotes the gradient operator for tensors, and $\underline{\underline{C}}=\underline{\underline{C}}(\mathbf{x})$ is the elasticity tensor. The components of $\underline{\underline{C}}$ are the elasticity moduli $C_{\mathrm{ijkl}}: \underline{\underline{C}}=\left(C_{\mathrm{ijkl}}(\mathbf{x})\right)$. The traction operator $\mathbf{T}$ is a surfacic differential operator defined on $\Gamma$ as

$$
\mathbf{T}(\mathbf{u})=\underline{\underline{\sigma}}(\mathbf{u}) \mathbf{n} .
$$

The right-hand side $\mathbf{f}$ is a data with support in $\Omega_{\mathrm{s}}$. The first transmission condition set on $\Gamma$ is a kinematic interface condition whereas the second one is a dynamic interface condition. The kinematic condition requires that the normal velocity of the fluid match the normal velocity of the solid on the interface $\Gamma$. The dynamic condition results from the equilibrium of forces on the interface $\Gamma$. The transmission conditions are natural.

Remark 2.1. We consider in this paper mainly Dirichlet external boundary conditions. In Appendix A from [23], we present also equivalent conditions up to the second order for the elato-acoustic problem complemented with a Fourier-Robin boundary condition set on $\Gamma^{\varepsilon}$.

In the framework above we address the issue of Equivalent Conditions (ECs) for the elastic displacement $\mathbf{u}_{\varepsilon}$ as $\varepsilon \rightarrow 0$, see Section 2.4. This issue is linked with the question of Uniform Estimates for the couple $\left(\mathbf{u}_{\varepsilon}, \mathbf{p}_{\varepsilon}\right)$ solution of the problem (2.1) as $\varepsilon \rightarrow 0$ (Sect. 2.5) since it is a main ingredient in the mathematical justification of ECs. To answer these questions, we make hereafter several assumptions on the data and on the regularity of the surface $\Gamma$. 


\subsection{Framework}

We will work under usual assumptions (symmetry and positiveness) on the elasticity tensor.

\section{Assumption 2.2.}

(i) The elasticity moduli $C_{\mathrm{ijkl}}(\mathbf{x})$ are real valued smooth functions in $\overline{\Omega_{\mathrm{s}}}$.

(ii) The tensor $\underline{\underline{C}}$ is symmetric:

$$
C_{\mathrm{ijkl}}=C_{\mathrm{jikl}}=C_{\mathrm{klij}} \quad \text { almost everywhere in } \Omega_{\mathrm{s}}
$$

(iii) The tensor $\underline{\underline{C}}$ is positive:

$$
\exists \alpha>0, \quad \forall \xi=\left(\xi_{\mathrm{ij}}\right) \text { symmetric tensor, } \sum_{i, j, k, l} C_{\mathrm{ijkl}} \xi_{\mathrm{ij}} \overline{\xi_{\mathrm{kl}}} \geqslant \alpha \sum_{i, j}\left|\xi_{\mathrm{ij}}\right|^{2} .
$$

Remark 2.3. According to the assumption 2.2(ii), the Hooke's law writes also $\underline{\underline{\sigma}}(\mathbf{u})=\underline{\underline{C}} \underline{\underline{\nabla}} \mathbf{u}$. Hence, the assumption 2.2(iii) ensures that the matrix differential operator $\nabla \cdot \underline{\underline{\sigma}}+\omega^{2} \rho \mathbb{I}$ is strongly elliptic.

Some resonant frequencies may appear in the solid domain. However, we prove uniform estimates for the elasto-acoustic field $\left(\mathbf{u}_{\varepsilon}, \mathbf{p}_{\varepsilon}\right)$ as well as ECs for $\mathbf{u}_{\varepsilon}$ when $\varepsilon \rightarrow 0$ under the following spectral assumption on the limit problem set in the solid part $\Omega_{\mathrm{s}}$, and when $\mathbf{f}=0$.

Assumption 2.4. The angular frequency $\omega$ is not an eigenfrequency of the problem

$$
\begin{cases}\nabla \cdot \underline{\sigma}(\mathbf{u})+\omega^{2} \rho \mathbf{u}=0 & \text { in } \Omega_{\mathrm{s}} \\ \mathbf{T}(\mathbf{u})=0 & \text { on } \Gamma .\end{cases}
$$

Our whole analysis is valid under the following assumption on the surfaces $\Gamma$ and $\Gamma_{\varepsilon}$.

Assumption 2.5. The fluid-solid interface $\Gamma$ and the surface $\Gamma_{\varepsilon}$ are smooth.

For the sake of simplicity in the asymptotic modeling, we will work under the following assumption on the data $\mathbf{f}$.

Assumption 2.6. The right-hand side $\mathbf{f}$ in (2.1) is a smooth $\varepsilon$-independent data.

In the framework above, we prove in this paper that it is possible to replace the fluid region $\Omega_{\mathrm{f}}^{\varepsilon}$ by appropriate boundary conditions called equivalent conditions and set on $\Gamma$.

\subsection{Validation of equivalent conditions}

In this paper, we derive surfacic differential operators $B_{\varepsilon}$

$$
\mathrm{B}_{\varepsilon}: \mathcal{C}^{\infty}(\Gamma) \rightarrow \mathcal{C}^{\infty}(\Gamma),
$$

together with $\tilde{\mathbf{u}}_{\varepsilon}$ which is a solution of the boundary value problem

$$
\begin{cases}\nabla \cdot \underline{\underline{\sigma}}\left(\tilde{\mathbf{u}}_{\varepsilon}\right)+\omega^{2} \rho \tilde{\mathbf{u}}_{\varepsilon}=\mathbf{f} & \text { in } \quad \Omega_{\mathbf{s}} \\ \mathbf{T}\left(\tilde{\mathbf{u}}_{\varepsilon}\right)+\mathrm{B}_{\varepsilon}\left(\tilde{\mathbf{u}}_{\varepsilon} \cdot \mathbf{n}\right) \mathbf{n}=0 & \text { on } \quad \Gamma\end{cases}
$$

Then in the framework of Section 2.3, we prove uniform estimates for the error between the exact solution $\mathbf{u}_{\varepsilon}$ in (2.1) and $\tilde{\mathbf{u}}_{\varepsilon}$ provided $\varepsilon$ is small enough:

$$
\left\|\mathbf{u}_{\varepsilon}-\tilde{\mathbf{u}}_{\varepsilon}\right\|_{1, \Omega_{\mathrm{s}}} \leqslant C \varepsilon^{k+1}
$$

with $k \in\{0,1,2,3\}$, see Theorem 3.4 for the main result and precise estimates. Here, we denote by $\|\cdot\|_{1, \Omega_{\mathrm{s}}}$ the norm in the Sobolev space $\mathbf{H}^{1}\left(\Omega_{\mathrm{s}}\right)=\mathrm{H}^{1}\left(\Omega_{\mathrm{s}}\right)^{3}$. We say that the equivalent condition is of order $k+1$ when such an a priori estimate (2.3) holds. Then we define $\mathbf{u}_{\varepsilon}^{k}=\tilde{\mathbf{u}}_{\varepsilon}$ and we denote by $\mathrm{B}_{k, \varepsilon}$ the operator $\mathrm{B}_{\varepsilon}$ corresponding to the order $k+1$, Section 3. The validation of ECs relies on uniform estimates for solutions $\left(\mathbf{u}_{\varepsilon}, \mathbf{p}_{\varepsilon}\right)$ of $(2.1)$ as $\varepsilon \rightarrow 0$. This issue is developped in Section 2.5. 


\subsection{Uniform estimates}

We introduce a suitable variational framework for the solution of the problem (2.1) with more general righthand sides. This framework is useful to prove error estimates (2.3).

\section{Weak solutions}

For given data $(\mathbf{f}, \mathrm{f}, \mathrm{g})$ we consider the boundary value problem

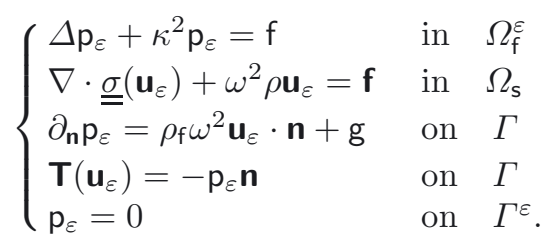

Hereafter, we explicit a weak formulation of the problem (2.4). We first introduce the functional space adapted to a variational formulation

$$
V_{\varepsilon}=\left\{(\mathbf{u}, \mathrm{p}) \in \mathbf{H}^{1}\left(\Omega_{\mathrm{s}}\right) \times \mathrm{H}^{1}\left(\Omega_{\mathrm{f}}^{\varepsilon}\right) \mid \gamma_{0} \mathrm{p}=0 \quad \text { on } \quad \Gamma^{\varepsilon}\right\} .
$$

Here, $\gamma_{0} \mathrm{p}$ is the Dirichlet trace of $\mathrm{p}$ on $\Gamma^{\varepsilon}$. The space $V_{\varepsilon}$ is endowed with the piecewise $\mathrm{H}^{1}$ norm in $\Omega_{\mathrm{s}}$ and $\Omega_{\mathrm{f}}^{\varepsilon}$. Then the variational problem writes: Find $\left(\mathbf{u}_{\varepsilon}, \mathrm{p}_{\varepsilon}\right) \in V_{\varepsilon}$ such that

$$
\forall(\mathbf{v}, \mathbf{q}) \in V_{\varepsilon}, \quad a_{\varepsilon}\left(\left(\mathbf{u}_{\varepsilon}, \mathbf{p}_{\varepsilon}\right),(\mathbf{v}, \mathbf{q})\right)=\langle F,(\mathbf{v}, \mathbf{q})\rangle_{V_{\varepsilon}^{\prime}, V_{\varepsilon}},
$$

where the sesquilinear form $a_{\varepsilon}$ is defined as

$$
a_{\varepsilon}((\mathbf{u}, \mathbf{p}),(\mathbf{v}, \mathbf{q}))=\int_{\Omega_{\mathrm{f}}^{\varepsilon}}\left(\nabla \mathbf{p} \cdot \nabla \overline{\mathbf{q}}-\kappa^{2} \mathbf{p} \overline{\mathbf{q}}\right) \mathrm{d} \mathbf{x}+\int_{\Omega_{\mathrm{s}}}\left(\underline{\underline{\sigma}}(\mathbf{u}): \underline{\underline{\epsilon}}(\overline{\mathbf{v}})-\omega^{2} \rho \mathbf{u} \overline{\mathbf{v}}\right) \mathrm{d} \mathbf{x}+\int_{\Gamma}\left(\omega^{2} \rho_{\mathrm{f}} \mathbf{u} \cdot \mathbf{n} \overline{\mathbf{q}}+\mathbf{p} \overline{\mathbf{v}} \cdot \mathbf{n}\right) \mathrm{d} \sigma
$$

and the right-hand side $F$ is defined as

$$
\langle F,(\mathbf{v}, \mathbf{q})\rangle_{V_{\varepsilon}^{\prime}, V_{\varepsilon}}=-\int_{\Omega_{\mathrm{f}}^{\varepsilon}} \mathrm{f} \overline{\mathrm{q}} \mathrm{d} \mathbf{x}-\int_{\Omega_{\mathrm{s}}} \mathbf{f} \cdot \overline{\mathbf{v}} \mathrm{d} \mathbf{x}-\int_{\Gamma} \mathrm{g} \overline{\mathrm{q}} \mathrm{d} \sigma .
$$

We assume that the data $(\mathbf{f}, \mathrm{f}, \mathrm{g})$ are smooth enough such that the right-hand side $F$ belongs to the space $V_{\varepsilon}^{\prime}$.

\section{Statement of uniform estimates}

In the framework of Section 2.3 we prove $\varepsilon$-uniform a priori estimates for the solution of problem (2.5). The following theorem is the main result in this section.

Theorem 2.7. Under Assumptions 2.2, 2.4, 2.5, there exists constants $\varepsilon_{0}, C>0$ such that for all $\varepsilon \in\left(0, \varepsilon_{0}\right)$, the problem (2.5) with data $F \in V_{\varepsilon}^{\prime}$ has a unique solution $\left(\mathbf{u}_{\varepsilon}, \mathrm{p}_{\varepsilon}\right) \in V_{\varepsilon}$ which satisfies

$$
\left\|\mathrm{p}_{\varepsilon}\right\|_{1, \Omega_{\mathrm{f}}^{\varepsilon}}+\left\|\mathbf{u}_{\varepsilon}\right\|_{1, \Omega_{\mathrm{s}}} \leqslant C\|F\|_{V_{\varepsilon}^{\prime}} .
$$

This result is proved in Section 4. The proof is based on a formulation of the problem set in a fixed domain. This formulation is obtained through a scaling along the thickness of the layer. As an application of uniform estimates (2.6), we prove the convergence result (2.3) in Section 6. 


\section{Equivalent conditions}

In the framework above, we derive for all $k \in\{0,1,2,3\}$ a boundary condition set on $\Gamma$ which is associated with the problem (2.1) and satisfied by $\mathbf{u}_{\varepsilon}^{k}$, i.e. $\mathbf{u}_{\varepsilon}^{k}$ solves the problem

$$
\begin{cases}\nabla \cdot \underline{\underline{\sigma}}\left(\mathbf{u}_{\varepsilon}^{k}\right)+\omega^{2} \rho \mathbf{u}_{\varepsilon}^{k}=\mathbf{f} & \text { in } \quad \Omega_{\mathrm{s}} \\ \mathbf{T}\left(\mathbf{u}_{\varepsilon}^{k}\right)+\mathrm{B}_{k, \varepsilon}\left(\mathbf{u}_{\varepsilon}^{k} \cdot \mathbf{n}\right) \mathbf{n}=0 & \text { on } \quad \Gamma .\end{cases}
$$

Here $\mathrm{B}_{k, \varepsilon}$ is a surfacic differential operator acting on functions defined on $\Gamma$ and which depends on $\varepsilon$. In this section, we present Equivalent Conditions (ECs) up to the fourth order and asymptotic models for the solution of the exact problem, Section 3.1. Then, we present well-posedness and convergence results, Section 3.2. Elements of derivation and mathematical validations for ECs are presented in Sections 5 and 6.

\subsection{Statement of equivalent conditions}

We obtain a hierarchy of boundary-value problems. Each one gives a model with a different order of accuracy in $\varepsilon$ and reflects the effect of the thin layer on the elastic displacement. We derive in Section 5.3 the following boundary conditions in problem (3.1):

Order 1

$$
\mathbf{T}\left(\mathbf{u}_{0}\right)=0 \quad \text { on } \quad \Gamma
$$

Order 2

$$
\mathbf{T}\left(\mathbf{u}_{\varepsilon}^{1}\right)-\varepsilon \omega^{2} \rho_{\mathbf{f}} \mathbf{u}_{\varepsilon}^{1} \cdot \mathbf{n} \mathbf{n}=0 \quad \text { on } \quad \Gamma
$$

Order 3

$$
\mathbf{T}\left(\mathbf{u}_{\varepsilon}^{2}\right)-\varepsilon \omega^{2} \rho_{\mathrm{f}}\left(1-\varepsilon \mathcal{H}\left(y_{\alpha}\right)\right) \mathbf{u}_{\varepsilon}^{2} \cdot \mathbf{n} \mathbf{n}=0 \quad \text { on } \quad \Gamma
$$

Order 4

$$
\mathbf{T}\left(\mathbf{u}_{\varepsilon}^{3}\right)-\varepsilon \omega^{2} \rho_{\mathrm{f}}\left(1-\varepsilon \mathcal{H}\left(y_{\alpha}\right)+\frac{\varepsilon^{2}}{3}\left[\Delta_{\Gamma}+\kappa^{2} \mathbb{I}+4 \mathcal{H}^{2}\left(y_{\alpha}\right)-\mathcal{K}\left(y_{\alpha}\right)\right]\right)\left(\mathbf{u}_{\varepsilon}^{3} \cdot \mathbf{n}\right) \mathbf{n}=0 \quad \text { on } \quad \Gamma
$$

Here, $\left(y_{\alpha}\right), \alpha=1,2$, are tangential coordinates on $\Gamma, \mathcal{H}$ and $\mathcal{K}$ denote the mean curvature and the Gaussian curvature of the surface $\Gamma$ and $\Delta_{\Gamma}$ is the Laplace-Beltrami operator along $\Gamma$. Successive corrections appear in these conditions when increasing the order of approximation. The conditions of order $k \in\{1,2,3\}$ involves only partial derivatives of order 1 in the operator $\mathbf{T}$, whereas the condition of order $k=4$ is a Ventcel's condition $[4,18]$ since it involves partial derivatives of order 2 .

Remark 3.1. The "background" solution $\mathbf{u}_{0}$ is independent of $\varepsilon$. It corresponds to a model where the thin layer is neglected. The effect of the fluid part appears from the order 2 through the density $\rho_{\mathrm{f}}$. The influence of the geometry of the surface $\Gamma$ appears from the order 3 through the mean curvature of $\Gamma$. The Helmholtz operator set on $\Gamma$ appears with the fourth order.

\section{Remark 3.2.}

(i) (Comparison with [6]). We have compared impedance operators for elasto-acoustics and acoustics [6] precisely. The comparison does not seem relevant. 
(ii) (Non-constant thickness). We consider here a thin layer with constant thickness. In the context of geophysical applications, the thickness of the layer is no longer constant with respect to the tangential variable. The change of variables (or scaling) would lead to additional terms in the transmission conditions. These terms come from the determinant of the metric of the layer. The derivation of the asymptotics are more tedious but all the tools are given in the present paper to perform the calculation.

\subsection{Stability and convergence of equivalent conditions}

Our goal in the next sections is to validate ECs set on $\Gamma$ (Sect. 3.1) proving estimates for $\mathbf{u}_{\varepsilon}-\mathbf{u}_{\varepsilon}^{k}$ for all $k \in\{0,1,2,3\}$, where $\mathbf{u}_{\varepsilon}^{k}$ is the solution of the approximate model (3.1), and $\mathbf{u}_{\varepsilon}$ satisfies the problem (2.1). The functional setting for $\mathbf{u}_{\varepsilon}^{k}$ is described by the Hilbert space $\mathbf{V}^{k}$ :

Notation 3.3. $\mathbf{V}^{k}$ denotes the space $\mathbf{H}^{1}\left(\Omega_{\mathrm{s}}\right)$ when $k \in\{0,1,2\}$, and $\left\{\mathbf{u} \in \mathbf{H}^{1}\left(\Omega_{\mathbf{s}}\right)|\mathbf{u} \cdot \mathbf{n}|_{\Gamma} \in \mathrm{H}^{1}(\Gamma)\right\}$ when $k=3$.

Theorem 3.4. Under Assumptions 2.2, 2.4, 2.5, 2.6, for all $k \in\{0,1,2,3\}$ there exists constants $\varepsilon_{k}, C_{k}>0$ such that for all $\varepsilon \in\left(0, \varepsilon_{k}\right)$, the problem (3.1) with data $\mathbf{f} \in \mathbf{L}^{2}\left(\Omega_{\mathbf{s}}\right)$ has a unique solution $\mathbf{u}_{\varepsilon}^{k} \in \mathbf{V}^{k}$ which satisfies uniform estimates

$$
\left\|\mathbf{u}_{\varepsilon}-\mathbf{u}_{\varepsilon}^{k}\right\|_{1, \Omega_{\mathrm{s}}} \leqslant C_{k} \varepsilon^{k+1} .
$$

The well-posedness result for the problem (3.1) is stated in Theorem 5.3 and is proved in Section 6.1. It appears nontrivial to work straightforwardly with the difference $\mathbf{u}_{\varepsilon}-\mathbf{u}_{\varepsilon}^{k}$. A usual method consists to use the truncated series $\mathbf{u}_{k, \varepsilon}$ introduced in Section 5.3 as intermediate quantities [13]. Then, the error analysis is splitted into two steps detailed in the next sections:

1. We prove uniform estimates for the difference $\mathbf{u}_{\varepsilon}-\mathbf{u}_{k, \varepsilon}$ in Theorem 5.2, Section 5.4,

2. We prove uniform estimates for the difference $\mathbf{u}_{k, \varepsilon}-\mathbf{u}_{\varepsilon}^{k}$, Section 6.2.

\section{UNIFORM ESTIMATES}

In this section, we prove uniform estimates for the exact solution of the elasto-acoustic problem. Since the functional setting of the variational problem (2.5) depends on the small parameter $\varepsilon$, it is not well suited to prove uniform estimates for solutions $\left(\mathbf{u}_{\varepsilon}, \mathbf{p}_{\varepsilon}\right) \in V_{\varepsilon}$. To overcome this difficulty, we adapt an idea developed in [6] writing equivalently the variational problem (2.5) in a common functional framework as $\varepsilon$ varying, Section 4.1. We state uniform estimates in this new framework, Theorem 4.1. We use a compactness argument to prove estimates, Section 4.2.

\subsection{The scaled problem}

We write the variational problem (2.5) in a fixed domain through the scaling $S=\varepsilon^{-1} \nu$ where $\nu \in(0, \varepsilon)$ is the distance to the surface $\Gamma$. The fixed domain writes $\Omega_{\mathrm{s}} \times \Omega_{\mathfrak{f}}$ where $\Omega_{\mathfrak{f}}:=\Gamma \times(0,1)$ and the ad-hoc functional space writes

$$
V=\left\{(\mathbf{u}, \mathfrak{p}) \in \mathbf{H}^{1}\left(\Omega_{\mathbf{s}}\right) \times \mathrm{H}^{1}\left(\Omega_{\mathfrak{f}}\right) \mid \quad \mathfrak{p}(., 1)=0 \quad \text { on } \quad \Gamma\right\} .
$$

Then the variational problem writes: Find $\left(\mathbf{u}_{\varepsilon}, \mathfrak{p}_{\varepsilon}\right) \in V$ such that for all $(\mathbf{v}, \mathfrak{q}) \in V$,

$$
\varepsilon a_{\mathfrak{f}}\left(\varepsilon ; \mathfrak{p}_{\varepsilon}, \mathfrak{q}\right)+a_{\mathbf{s}}\left(\mathbf{u}_{\varepsilon}, \mathbf{v}\right)+\int_{\Gamma}\left(\omega^{2} \rho_{\mathbf{f}} \mathbf{u} \cdot \mathbf{n} \overline{\mathfrak{q}}+\mathfrak{p} \overline{\mathbf{v}} \cdot \mathbf{n}\right) \mathrm{d} \Gamma=\left\langle\mathfrak{F}_{\varepsilon},(\mathbf{v}, \mathfrak{q})\right\rangle_{V^{\prime}, V},
$$

where

$$
a_{\mathfrak{f}}(\varepsilon ; \mathfrak{p}, \mathfrak{q})=\int_{0}^{1} \int_{\Gamma}\left\{(\mathbb{I}+\varepsilon S \mathcal{R})^{-2} \nabla_{\Gamma} \mathfrak{p} \nabla_{\Gamma} \overline{\mathfrak{q}}+\varepsilon^{-2} \partial_{S} \mathfrak{p} \partial_{S} \overline{\mathfrak{q}}-\kappa^{2} \mathfrak{p} \overline{\mathfrak{q}}\right\} \operatorname{det}(\mathbb{I}+\varepsilon S \mathcal{R}) \mathrm{d} \Gamma \mathrm{d} S
$$




$$
a_{\mathbf{s}}(\mathbf{u}, \mathbf{v})=\int_{\Omega_{\mathrm{s}}}\left(\underline{\underline{\sigma}}(\mathbf{u}): \underline{\underline{\epsilon}}(\overline{\mathbf{v}})-\omega^{2} \rho \mathbf{u} \overline{\mathbf{v}}\right) \mathrm{d} \mathbf{x},
$$

$$
\text { and }\left\langle\mathfrak{F}_{\varepsilon},(\mathbf{v}, \mathfrak{q})\right\rangle_{V^{\prime}, V}=-\varepsilon \int_{\Omega_{\mathfrak{f}}} \mathfrak{f} \overline{\mathfrak{q}} \operatorname{det}(\mathbb{I}+\varepsilon S \mathcal{R}) \mathrm{d} \Gamma \mathrm{d} S-\int_{\Omega_{\mathrm{s}}} \mathbf{f} \cdot \overline{\mathbf{v}} \mathrm{d} \mathbf{x}-\int_{\Gamma} \mathfrak{g} \overline{\mathfrak{q}} \mathrm{d} \Gamma
$$

Here $\mathcal{R}$ is an intrinsic symmetric linear operator defined on the tangent plane $\mathbf{T}_{\mathbf{x}_{\Gamma}}(\Gamma)$ to $\Gamma$ at the point $\mathbf{x}_{\Gamma} \in \Gamma$ which characterizes the curvature of $\Gamma$ at the point $\mathbf{x}_{\Gamma}$. We refer to [6] and Section 4.1 from [23] for the introduction of geometrical tools and more details. The parameter $\varepsilon$ weighting the form $a_{\mathfrak{f}}(\varepsilon ; \mathfrak{p}, \mathfrak{q})$ in formulation (4.1) may lead to a solution $\left(\mathbf{u}_{\varepsilon}, \mathfrak{p}_{\varepsilon}\right) \in V$ such that the surface gradient $\nabla_{\Gamma} \mathfrak{p}_{\varepsilon}$ can be unbounded as $\varepsilon \rightarrow 0$. This is a similarity with the work in Reference [6]. Furthermore, the sign of the left-hand side of the problem (4.1) for $(\mathbf{v}, \mathfrak{q})=\left(\mathbf{u}_{\varepsilon}, \mathfrak{p}_{\varepsilon}\right)$ cannot be controlled. Hence due to the lack of strong coerciveness of the variational formulation (4.1) one cannot get straightforwardly estimates. Our main result for the problem (4.1) is the following a priori estimate, uniform as $\varepsilon \rightarrow 0$.

Theorem 4.1. Under Assumptions 2.2, 2.4, 2.5, there exists constants $\varepsilon_{0}, C>0$ such that for all $\varepsilon \in\left(0, \varepsilon_{0}\right)$, the problem (4.1) with data $\mathfrak{F}_{\varepsilon} \in V^{\prime}$ has a unique solution $\left(\mathbf{u}_{\varepsilon}, \mathfrak{p}_{\varepsilon}\right) \in V$ which satisfies the uniform estimates

$$
\sqrt{\varepsilon}\left\|\nabla_{\Gamma} \mathfrak{p}_{\varepsilon}\right\|_{0, \Omega_{\mathfrak{f}}}+\sqrt{\varepsilon}^{-1}\left\|\partial_{S} \mathfrak{p}_{\varepsilon}\right\|_{0, \Omega_{\mathfrak{f}}}+\left\|\mathfrak{p}_{\varepsilon}\right\|_{0, \Omega_{\mathfrak{f}}}+\left\|\mathfrak{p}_{\varepsilon}\right\|_{0, \Gamma}+\left\|\mathbf{u}_{\varepsilon}\right\|_{1, \Omega_{\mathrm{s}}} \leqslant C\left\|\mathfrak{F}_{\varepsilon}\right\|_{V^{\prime}}
$$

This theorem is the key for the proof of Theorem 2.7: as a consequence of the following estimates

$$
\begin{gathered}
\forall \mathrm{p} \in \mathrm{L}^{2}\left(\Omega_{\mathrm{f}}^{\varepsilon}\right), \quad\|\mathrm{p}\|_{0, \Omega_{\mathrm{f}}^{\varepsilon}} \simeq \sqrt{\varepsilon}\|\mathfrak{p}\|_{0, \Omega_{\mathfrak{f}}}, \\
\forall \mathrm{p} \in \mathrm{H}^{1}\left(\Omega_{\mathrm{f}}^{\varepsilon}\right), \quad\|\nabla \mathrm{p}\|_{0, \Omega_{\mathrm{f}}^{\varepsilon}} \simeq \sqrt{\varepsilon}\left\|\nabla_{\Gamma} \mathfrak{p}\right\|_{0, \Omega_{\mathfrak{f}}}+\sqrt{\varepsilon}-1\left\|\partial_{S} \mathfrak{p}\right\|_{0, \Omega_{\mathrm{f}}},
\end{gathered}
$$

available for $\varepsilon$ small enough, and a proof of which can be found in ([23], Sect. 4.1), we obtain estimates (2.6). In (4.3a), (4.3b), for any function $\mathrm{p}$ defined in $\Omega_{\mathfrak{f}}^{\varepsilon}$, the function $\mathfrak{p}$ is defined in the domain $\Omega_{\mathfrak{f}}$ as

$$
\mathfrak{p}\left(\mathbf{x}_{\Gamma}, S\right)=\mathrm{p}(\mathbf{x}), \quad\left(\mathbf{x}_{\Gamma}, S=\frac{\nu}{\varepsilon}\right) \in \Gamma \times(0,1) .
$$

In (4.3a), the symbol $\simeq$ means that quantities $\|\mathrm{p}\|_{0, \Omega_{\mathfrak{f}}^{\varepsilon}}$ and $\sqrt{\varepsilon}\|\mathfrak{p}\|_{0, \Omega_{\mathfrak{f}}}$ are equal up to a multiplicative constant which is independent of $\varepsilon$.

The proof of Theorem 4.1 is based on the following statement.

Lemma 4.2. Under Assumptions 2.2, 2.4, 2.5, there exists constants $\varepsilon_{0}, C>0$ such that for all $\varepsilon \in\left(0, \varepsilon_{0}\right)$, any solution $\left(\mathbf{u}_{\varepsilon}, \mathfrak{p}_{\varepsilon}\right) \in V$ of problem (4.1) with a data $\mathfrak{F}_{\varepsilon} \in V^{\prime}$ satisfies the uniform estimates

$$
\left\|\left(\mathbf{u}_{\varepsilon}, \mathfrak{p}_{\varepsilon}\right)\right\|_{0, \Omega_{\mathrm{s}} \times \Omega_{\mathrm{f}}}+\left\|\mathbf{u}_{\varepsilon} \cdot \mathbf{n}\right\|_{0, \Gamma}+\left\|\mathfrak{p}_{\varepsilon}\right\|_{0, \Gamma} \leqslant C\left\|\mathfrak{F}_{\varepsilon}\right\|_{V^{\prime}} .
$$

This Lemma is going to be proved in Section 4.2. The proof of this result involves both a compactness argument and the spectral assumption 2.4. As a consequence of estimates (4.4), we infer estimates (4.2). Since the problem (4.1) is of Fredholm type, Theorem 4.1 is then obtained as a consequence of the Fredholm alternative.

\subsection{Proof of Lemma 4.2: Uniform estimate of $\left(u_{\varepsilon}, \mathfrak{p}_{\varepsilon}\right)$}

We prove this lemma by contradiction: We assume that there exists a sequence $\left(\mathbf{u}_{m}, \mathfrak{p}_{m}\right) \in V, m \in \mathbb{N}$, of solutions of problem (4.1) associated with a parameter $\varepsilon_{m}$ and a right-hand side $\mathfrak{F}_{m} \in V^{\prime}$ :

$$
\forall(\mathbf{v}, \mathfrak{q}) \in V, \quad \varepsilon_{m} a_{\mathfrak{f}}\left(\varepsilon_{m} ; \mathfrak{p}_{m}, \mathfrak{q}\right)+a_{\mathbf{s}}\left(\mathbf{u}_{m}, \mathbf{v}\right)+\int_{\Gamma}\left(\omega^{2} \rho_{\mathfrak{f}} \mathbf{u}_{m} \cdot \mathbf{n} \overline{\mathfrak{q}}+\mathfrak{p}_{m} \overline{\mathbf{v}} \cdot \mathbf{n}\right) \mathrm{d} \Gamma=\left\langle\mathfrak{F}_{m},(\mathbf{v}, \mathfrak{q})\right\rangle_{V^{\prime}, V},
$$


satisfying the following conditions

$$
\begin{array}{cl}
\varepsilon_{m} \rightarrow 0 & \text { as } m \rightarrow \infty, \\
\left\|\left(\mathbf{u}_{m}, \mathfrak{p}_{m}\right)\right\|_{0, \Omega_{\mathrm{s}} \times \Omega_{\mathrm{f}}}+\left\|\mathbf{u}_{m} \cdot \mathbf{n}\right\|_{0, \Gamma}+\left\|\mathfrak{p}_{m}\right\|_{0, \Gamma}=1 & \text { for all } m \in \mathbb{N}, \\
\left\|\mathfrak{F}_{m}\right\|_{V^{\prime}} \rightarrow 0 & \text { as } m \rightarrow \infty .
\end{array}
$$

Choosing tests functions $(\mathbf{v}, \mathfrak{q})=\left(\mathbf{u}_{m}, \mathfrak{p}_{m}\right)$ in (4.5), we obtain with the help of conditions (4.6a) $-(4.6 \mathrm{c})$ the following uniform bounds:

(i) The sequence $\left\{\mathbf{u}_{m}, \mathfrak{p}_{m}\right\}$ is bounded in the space $W$ defined as

$$
\begin{gathered}
W=\left\{(\mathbf{u}, \mathfrak{p}) \in \mathbf{H}^{1}\left(\Omega_{\mathbf{s}}\right) \times \mathrm{H}^{1}\left(0,1 ; \mathrm{L}^{2}(\Gamma)\right) \mid \mathfrak{p}(., 1)=0 \quad \text { on } \quad \Gamma\right\}, \\
\left\|\left(\mathbf{u}_{m}, \mathfrak{p}_{m}\right)\right\|_{W} \leqslant C .
\end{gathered}
$$

Remind that $\mathrm{H}^{1}\left(0,1 ; \mathrm{L}^{2}(\Gamma)\right)$ is the space of distributions $\mathfrak{p} \in \mathcal{D}^{\prime}\left(0,1 ; \mathrm{L}^{2}(\Gamma)\right)$ such that $\mathfrak{p}$ and $\mathfrak{p}^{\prime}$ belong to $\mathrm{L}^{2}\left(0,1 ; \mathrm{L}^{2}(\Gamma)\right)$. Subsequently, we identify the space $\mathrm{L}^{2}\left(0,1 ; \mathrm{L}^{2}(\Gamma)\right)$ and $\mathrm{L}^{2}\left(\Omega_{\mathrm{f}}\right)$.

Since the domain $\Omega_{\mathrm{s}}$ is bounded, the embedding of $\mathbf{H}^{1}\left(\Omega_{\mathrm{s}}\right)$ into $\mathbf{L}^{2}\left(\Omega_{\mathrm{s}}\right)$ is compact. Hence as a consequence of (4.7), using the Rellich Lemma we can extract a subsequence of $\left\{\mathbf{u}_{m}, \mathfrak{p}_{m}\right\}$ (still denoted by $\left\{\mathbf{u}_{m}, \mathfrak{p}_{m}\right\}$ ) which is converging in $\mathbf{L}^{2}\left(\Omega_{\mathbf{s}}\right) \times L^{2}\left(\Omega_{\mathfrak{f}}\right)$, and we can assume that the sequence $\left\{\underline{\underline{\nabla}} \mathbf{u}_{m}\right\}$ is weakly converging in $\mathbf{L}^{2}\left(\Omega_{\mathbf{s}}\right)$.

(ii) The sequence $\left\{{\sqrt{\varepsilon_{m}}}^{-1} \partial_{S} \mathfrak{p}_{m}\right\}$ is bounded in $\mathrm{L}^{2}\left(\Omega_{\mathfrak{f}}\right)$, hence the sequence $\left\{\partial_{S} \mathfrak{p}_{m}\right\}$ converges to 0 in $L^{2}\left(\Omega_{\mathfrak{f}}\right)$.

\section{Limit of the sequences $\left\{\mathbf{u}_{m}, \mathfrak{p}_{m}\right\}$}

To prove convergence results for $\left\{\mathbf{u}_{m}, \mathfrak{p}_{m}\right\}$, we use Poincaré and trace inequalities which are available in the Hilbert space

$$
\mathrm{H}=\left\{\mathfrak{p} \in \mathrm{H}^{1}\left(0,1 ; \mathrm{L}^{2}(\Gamma)\right) \mid \mathfrak{p}(., 1)=0 \quad \text { on } \quad \Gamma\right\},
$$

see ([23], Prop. 4.4, 4.5): There exists $C_{P}, C>0$ such that

$$
\forall \mathfrak{p} \in \mathrm{H}, \quad\|\mathfrak{p}\|_{0, \Omega_{\mathfrak{f}}} \leqslant C_{P}\left\|\partial_{S} \mathfrak{p}\right\|_{0, \Omega_{\mathfrak{f}}} \quad \text { and } \quad\left\|\gamma_{0} \mathfrak{p}\right\|_{0, \Gamma} \leqslant C\left\|\partial_{S} \mathfrak{p}\right\|_{0, \Omega_{\mathfrak{f}}}
$$

As a consequence of (ii) and (4.8), we infer that sequences $\left\{\mathfrak{p}_{m}\right\}$ and $\left\{\gamma_{0} \mathfrak{p}_{m}\right\}$ converge to 0 in $L^{2}\left(\Omega_{\mathfrak{f}}\right)$ and to 0 in $\mathrm{L}^{2}(\Gamma)$ respectively

$$
\left\{\begin{array}{lll}
\mathfrak{p}_{m} \rightarrow 0 & \text { in } & \mathrm{L}^{2}\left(\Omega_{\mathfrak{f}}\right) \\
\gamma_{0} \mathfrak{p}_{m} \rightarrow 0 & \text { in } & \mathrm{L}^{2}(\Gamma)
\end{array}\right.
$$

Another consequence of (4.7) is that the sequence $\left\{\mathbf{u}_{m} \cdot \mathbf{n}\right\}$ is bounded in $\mathrm{H}^{\frac{1}{2}}(\Gamma)$. Therefore (up to the extraction of a subsequence) we can assume that the sequence $\left\{\mathbf{u}_{m} \cdot \mathbf{n}\right\}$ is strongly converging in $\mathrm{L}^{2}(\Gamma)$. Summarizing these convergence results, we deduce that there exists $\mathbf{u} \in \mathbf{L}^{2}\left(\Omega_{\mathbf{s}}\right)$ such that

$$
\left\{\begin{array}{lll}
\underline{\epsilon}\left(\mathbf{u}_{m}\right) \rightarrow \underline{\epsilon}(\mathbf{u}) & \text { in } & \mathbf{L}^{2}\left(\Omega_{\mathrm{s}}\right) \\
\mathbf{u}_{m} \rightarrow \mathbf{u} & \text { in } & \mathbf{L}^{2}\left(\Omega_{\mathrm{s}}\right) \\
\mathbf{u}_{m} \cdot \mathbf{n} \rightarrow \mathbf{u} \cdot \mathbf{n} & \text { in } & \mathrm{L}^{2}(\Gamma) .
\end{array}\right.
$$

As a consequence of the strong convergence of sequences $\left\{\mathbf{u}_{m}\right\}$ in $\mathbf{L}^{2}\left(\Omega_{\mathbf{s}}\right)$ and $\left\{\mathbf{u}_{m} \cdot \mathbf{n}\right\}$ in $\mathrm{L}^{2}(\Gamma)$, and the strong convergence of $\mathfrak{p}_{m}$ and $\gamma_{0} \mathfrak{p}_{m}$ (4.9) together with (4.6b), we infer

$$
\|\mathbf{u}\|_{0, \Omega_{\mathrm{s}}}+\|\mathbf{u} \cdot \mathbf{n}\|_{0, \Gamma}=1
$$




\subsubsection{Conclusion}

Using Assumption 2.4, we are going to prove hereafter that $\mathbf{u}=0$, which will contradict $\|\mathbf{u}\|_{0, \Omega_{\mathrm{s}}}+\|\mathbf{u} \cdot \mathbf{n}\|_{0, \Gamma}=1$, and finally prove estimate (4.4). We use $(\mathbf{v}, \mathfrak{q}=0) \in V$ as test functions in (4.5): there holds

$$
\int_{\Omega_{\mathrm{s}}}\left(\underline{\underline{\sigma}}\left(\mathbf{u}_{m}\right): \underline{\underline{\epsilon}}(\overline{\mathbf{v}})-\omega^{2} \rho \mathbf{u}_{m} \overline{\mathbf{v}}\right) \mathrm{d} \mathbf{x}+\int_{\Gamma} \mathfrak{p}_{m} \overline{\mathbf{v}} \cdot \mathbf{n} \mathrm{d} \Gamma=\left\langle\mathfrak{F}_{m},(\mathbf{v}, 0)\right\rangle_{V^{\prime}, V} .
$$

According to (4.10), (4.9) and (4.6c), taking limits as $m \rightarrow+\infty$, we deduce from the previous equalities $\mathbf{u} \in \mathbf{H}^{1}\left(\Omega_{\mathrm{s}}\right)$ satisfies for all $\mathbf{v} \in \mathbf{H}^{1}\left(\Omega_{\mathrm{s}}\right)$ :

$$
\int_{\Omega_{\mathrm{s}}}\left(\underline{\underline{\sigma}}(\mathbf{u}): \underline{\underline{\epsilon}}(\overline{\mathbf{v}})-\omega^{2} \rho \mathbf{u} \overline{\mathbf{v}}\right) \mathrm{d} \mathbf{x}=0 .
$$

Integrating by parts the first term in the sesquilinear form (4.11), we find that $\mathbf{u}$ satisfies the problem

$$
\left\{\begin{array}{lll}
\nabla \cdot \underline{\sigma}(\mathbf{u})+\omega^{2} \rho \mathbf{u}=0 & \text { in } & \Omega_{\mathbf{s}} \\
\mathbf{T}(\mathbf{u})=0 & \text { on } & \Gamma
\end{array}\right.
$$

By Assumption 2.4, we deduce

$$
\mathbf{u}=0 \quad \text { in } \Omega_{\mathrm{s}},
$$

which contradicts $\|\mathbf{u}\|_{0, \Omega}+\|\mathbf{u} \cdot \mathbf{n}\|_{0, \Gamma}=1$ and ends the proof of Lemma 4.2.

\section{Derivation of Equivalent CONDitions}

In this section, we exhibit an asymptotic expansion for $\mathbf{u}_{\varepsilon}$ and $\mathbf{p}_{\varepsilon}$, Section 5.1. We explicit the first terms in asymptotics, Section 5.2. Then we construct formally equivalent conditions, Section 5.3. In Section 5.4 we validate the asymptotic expansion with estimates for the remainders. The main result of this section is the Theorem 5.3 in Section 5.5 which proves the stability of equivalent conditions.

\subsection{Multiscale expansion}

We can exhibit series expansions in powers of $\varepsilon$ for $\mathbf{u}_{\varepsilon}$ and $\mathrm{p}_{\varepsilon}$ :

$$
\begin{gathered}
\mathbf{u}_{\varepsilon}(\mathbf{x}) \approx \sum_{j \geqslant 0} \varepsilon^{j} \mathbf{u}_{j}(\mathbf{x}), \\
\mathrm{p}_{\varepsilon}(\mathbf{x}) \approx \sum_{j \geqslant 0} \varepsilon^{j} \mathbf{p}_{j}(\mathbf{x} ; \varepsilon) \quad \text { with } \quad \mathbf{p}_{j}(\mathbf{x} ; \varepsilon)=\mathfrak{p}_{j}\left(y_{\alpha}, \frac{y_{3}}{\varepsilon}\right),
\end{gathered}
$$

see Section 5.4 for precise estimates. Here $\left(y_{\alpha}, y_{3}\right)$ is a "normal coordinate system" $[8,23]$ to the surface $\Gamma$ on the manifold $\Omega_{\mathrm{f}}^{\varepsilon}: y_{\alpha}(\alpha \in\{1,2\})$ is a tangential coordinate on $\Gamma$ and $y_{3} \in(0, \varepsilon)$ is the distance to the surface $\Gamma$. The term $\mathfrak{p}_{j}$ is a "profile" defined on $\Gamma \times(0,1)$. The formal calculus concerning the problem are presented in Section 5.1.1 and the first terms $\left(\mathfrak{p}_{j}, \mathbf{u}_{j}\right)$ for $j=0,1,2,3$ are explicited in Section 5.2.

Expansion of the helmholtz operator

It is possible to write the three dimensional Helmholtz operator in the layer $\Omega_{f}^{\varepsilon}$ through the local coordinates $\left(y_{\alpha}, y_{3}\right)$ ([23], Prop. B.1). Then we make the scaling $Y_{3}=\varepsilon^{-1} y_{3} \in(0,1)$ into the normal coordinate and we expand formally the Helmholtz operator in power series of $\varepsilon$ with coefficient intrinsic operators:

$$
\Delta+\kappa^{2} \mathrm{Id}=\varepsilon^{-2}\left(\sum_{n=0}^{N-1} \varepsilon^{n} \mathrm{~L}^{n}+\varepsilon^{N} \mathrm{R}_{\varepsilon}^{N}\right) \quad \text { for all } \quad N \in \mathbb{N}^{*} .
$$


The operators $\mathrm{L}^{n}, n=0,1,2$, are explicited in Proposition B.3 from [23]:

$$
\mathrm{L}^{0}=\partial_{3}^{2}, \quad \mathrm{~L}^{1}=2 \mathcal{H}\left(y_{\alpha}\right) \partial_{3}, \quad \text { and } \quad \mathrm{L}^{2}=\Delta_{\Gamma}+\kappa^{2} \mathbb{I}-2\left(2 \mathcal{H}^{2}-\mathcal{K}\right)\left(y_{\alpha}\right) Y_{3} \partial_{3} .
$$

Here $\partial_{3}$ is the partial derivative with respect to $Y_{3}$. We remind that $\Delta_{\Gamma}$ is the Laplace-Beltrami operator along $\Gamma, \mathcal{H}$ and $\mathcal{K}$ are the mean and the Gaussian curvature of the surface $\Gamma$. The remainder $\mathrm{R}_{\varepsilon}^{N}$ has smooth coefficients in $y_{\alpha}$ and $Y_{3}$ which are bounded in $\varepsilon$.

\subsubsection{Elementary problems}

After the change of variables $y_{3} \mapsto Y_{3}=\varepsilon^{-1} y_{3}$ in the thin layer $\Omega_{\mathrm{f}}^{\varepsilon}$, problem (2.1) becomes:

$$
\begin{cases}\varepsilon^{-2}\left[\partial_{3}^{2} \mathfrak{p}_{\varepsilon}+\sum_{n \geqslant 1} \varepsilon^{n} L^{n} \mathfrak{p}_{\varepsilon}\right]=0 & \text { in } \Gamma \times(0,1) \\ \varepsilon^{-1} \partial_{3} \mathfrak{p}_{\varepsilon}=\rho_{\mathrm{f}} \omega^{2} \mathbf{u}_{\varepsilon} \cdot \mathbf{n} & \text { on } \Gamma \times\{0\} \\ \mathfrak{p}_{\varepsilon}=0 & \text { on } \Gamma \times\{1\} \\ \nabla \cdot \underline{\sigma}\left(\mathbf{u}_{\varepsilon}\right)+\omega^{2} \rho \mathbf{u}_{\varepsilon}=\mathbf{f} & \text { in } \Omega_{\mathbf{s}} \\ \mathbf{T}\left(\mathbf{u}_{\varepsilon}\right)=-\mathfrak{p}_{\varepsilon} \mathbf{n} & \text { on } \Gamma .\end{cases}
$$

Inserting the Ansatz (5.1)-(5.2) in equations (5.3), we get the following two families of problems, coupled by their boundary conditions on $\Gamma$ (i.e. when $\left.Y_{3}=0\right)$ :

$$
\begin{gathered}
\left\{\begin{array}{lll}
\partial_{3}^{2} \mathfrak{p}_{n}=-\sum_{l+m=n, l \geqslant 1} L^{l} \mathfrak{p}_{m} & \text { for } & Y_{3} \in(0,1) \\
\partial_{3} \mathfrak{p}_{n}=\rho_{\mathbf{f}} \omega^{2} \mathbf{u}_{n-1} \cdot \mathbf{n} & \text { for } & Y_{3}=0 \\
\mathfrak{p}_{n}=0 & \text { for } & Y_{3}=1
\end{array}\right. \\
\begin{cases}\nabla \cdot \underline{\sigma}\left(\mathbf{u}_{n}\right)+\omega^{2} \rho \mathbf{u}_{n}=\mathbf{f} \delta_{0}^{n} & \text { in } \Omega_{\mathbf{s}} \\
\mathbf{T}\left(\mathbf{u}_{n}\right)=-\mathfrak{p}_{n} \mathbf{n} & \text { on } \quad \Gamma .\end{cases}
\end{gathered}
$$

In (5.4), we use the convention $\mathbf{u}_{-1}=0$, and in (5.5) $\delta_{0}^{n}$ denotes the Kronecker symbol.

\subsection{First terms}

We find successively from (5.4) $-(5.5)$ when $n=0,1,2,3$

$$
\begin{aligned}
& \mathfrak{p}_{0}=0, \\
& \begin{cases}\nabla \cdot \underline{\underline{\sigma}}\left(\mathbf{u}_{0}\right)+\omega^{2} \rho \mathbf{u}_{0}=\mathbf{f} & \text { in } \Omega_{\mathbf{s}} \\
\mathbf{T}\left(\mathbf{u}_{0}\right)=0 & \text { on } \Gamma,\end{cases} \\
& \mathfrak{p}_{1}\left(., Y_{3}\right)=\left.\left(Y_{3}-1\right) \rho_{\mathrm{f}} \omega^{2} \mathbf{u}_{0} \cdot \mathbf{n}\right|_{\Gamma}, \\
& \begin{cases}\nabla \cdot \underline{\underline{\sigma}}\left(\mathbf{u}_{1}\right)+\omega^{2} \rho \mathbf{u}_{1}=0 & \text { in } \Omega_{\mathbf{s}} \\
\mathbf{T}\left(\mathbf{u}_{1}\right)=\rho_{\mathrm{f}} \omega^{2} \mathbf{u}_{0} \cdot \mathbf{n} \mathbf{n} & \text { on } \Gamma,\end{cases} \\
& \mathfrak{p}_{2}\left(., Y_{3}\right)=-\left.\left(Y_{3}^{2}-1\right) \mathcal{H} \rho_{\mathrm{f}} \omega^{2} \mathbf{u}_{0} \cdot \mathbf{n}\right|_{\Gamma}+\left.\left(Y_{3}-1\right) \rho_{\mathrm{f}} \omega^{2} \mathbf{u}_{1} \cdot \mathbf{n}\right|_{\Gamma}, \\
& \begin{cases}\nabla \cdot \underline{\sigma}\left(\mathbf{u}_{2}\right)+\omega^{2} \rho \mathbf{u}_{2}=0 & \text { in } \Omega_{\mathrm{s}} \\
\mathbf{T}\left(\underline{\mathbf{u}_{2}}\right)=\rho_{\mathrm{f}} \omega^{2}\left(\mathbf{u}_{1}-\mathcal{H}\left(\mathbf{u}_{0}\right) \cdot \mathbf{n} \mathbf{n}\right. & \text { on } \Gamma,\end{cases}
\end{aligned}
$$




$$
\begin{aligned}
& \mathfrak{p}_{3}\left(., Y_{3}\right)=\left(Y_{3}^{3}-1\right) \frac{1}{6} \rho_{\mathrm{f}} \omega^{2}\left(8 \mathcal{H}^{2}-2 \mathcal{K}-\left(\Delta_{\Gamma}+\kappa^{2} \mathbb{I}\right)\right)\left(\mathbf{u}_{0} \cdot \mathbf{n}\right) \\
& \quad+\left(Y_{3}^{2}-1\right) \frac{1}{2} \rho_{\mathrm{f}} \omega^{2}\left(\left(\Delta_{\Gamma}+\kappa^{2} \mathbb{I}\right)\left(\mathbf{u}_{0} \cdot \mathbf{n}\right)-2 \mathcal{H} \mathbf{u}_{1} \cdot \mathbf{n}\right)+\left(Y_{3}-1\right) \rho_{\mathrm{f}} \omega^{2} \mathbf{u}_{2} \cdot \mathbf{n}, \\
& \left\{\begin{array}{l}
\nabla \cdot \underline{\underline{\sigma}}\left(\mathbf{u}_{3}\right)+\omega^{2} \rho \mathbf{u}_{3}=0 \text { in } \Omega_{\mathrm{s}} \\
\mathbf{T}\left(\mathbf{u}_{3}\right)=\rho_{\mathrm{f}} \omega^{2}\left(\frac{1}{3}\left(4 \mathcal{H}^{2}-\mathcal{K}+\Delta_{\Gamma}+\kappa^{2} \mathbb{I}\right)\left(\mathbf{u}_{0} \cdot \mathbf{n}\right)+\left(-\mathcal{H} \mathbf{u}_{1}+\mathbf{u}_{2}\right) \cdot \mathbf{n}\right) \mathbf{n} \quad \text { on } \Gamma .
\end{array}\right.
\end{aligned}
$$

We refer the reader to [23] for more details. The whole construction of the asymptotics comes from a recurrence argument: if the sequences $\left(\mathbf{u}_{n}\right)$ and $\left(\mathfrak{p}_{n}\right)$ are known until rank $n=N-1$, then the Sturm-Liouville problem (5.4) uniquely defines $\mathfrak{p}_{N}$ whose trace on $\Gamma$ is inserted into (5.5) as a data to determine $\mathbf{u}_{N}$. The next proposition ensures regularity results for the first terms $\mathbf{u}_{k}$ in $\mathbf{V}^{k}$ (Not. 3.3) $k=0,1,2,3$.

Proposition 5.1. Under Assumptions 2.2, 2.4, 2.5, if $\mathbf{f} \in \mathbf{L}^{2}\left(\Omega_{\mathrm{s}}\right)$ then elementary problems (5.6)-(5.7)-(5.9)-(5.11) have a unique solution $\mathbf{u}_{k}$ in $\mathbf{V}^{k}$ when $k=0,1,2,3$.

Let $l$ be a non-negative integer. If $\Omega_{\mathbf{s}}$ is of class $\mathcal{C}^{l+2}$ and $\mathbf{f} \in \mathbf{H}^{l}\left(\Omega_{\mathbf{s}}\right)$, then $\mathbf{u}_{0}, \mathbf{u}_{3}$ belong to $\mathbf{H}^{l+2}\left(\Omega_{\mathbf{s}}\right)$ and $\mathbf{u}_{1}, \mathbf{u}_{2}$ belong to $\mathbf{H}^{l+3}\left(\Omega_{\mathbf{s}}\right)$.

Here, the regularity of $\mathbf{u}_{0}, \mathbf{u}_{1}, \mathbf{u}_{2}$ and $\mathbf{u}_{3}$ is a consequence of a general shift result available in Sobolev spaces ([9], Thm. 3.4.5), since $\left.\mathfrak{p}_{1}\right|_{\Gamma} \in \mathrm{H}^{l+\frac{3}{2}}(\Gamma),\left.\mathfrak{p}_{2}\right|_{\Gamma} \in \mathrm{H}^{l+\frac{3}{2}}(\Gamma)$ and $\left.\mathfrak{p}_{3}\right|_{\Gamma} \in \mathrm{H}^{l+\frac{1}{2}}(\Gamma)$.

\subsection{Construction of equivalent conditions}

In this section, we derive formally ECs (Sect. 3.1).

\section{Order 1}

Since the equations in (5.6) are independent of $\varepsilon$, the condition of order 1 is the stress free boundary condition

$$
\mathbf{T}\left(\mathbf{u}_{0}\right)=0 \quad \text { on } \quad \Gamma .
$$

Order 2

According to (5.6) and (5.7), the truncated expansion $\mathbf{u}_{1, \varepsilon}=\mathbf{u}_{0}+\varepsilon \mathbf{u}_{1}$ solves the elastic equation in $\Omega_{\mathrm{s}}$ together with the boundary condition

$$
\mathbf{T}\left(\mathbf{u}_{1, \varepsilon}\right)=\varepsilon \rho_{\mathrm{f}} \omega^{2} \mathbf{u}_{0} \cdot \mathbf{n} \mathbf{n} \quad \text { on } \quad \Gamma .
$$

Writting $\mathbf{u}_{0}=\mathbf{u}_{1, \varepsilon}-\varepsilon \mathbf{u}_{1}$, there holds

$$
\mathbf{T}\left(\mathbf{u}_{1, \varepsilon}\right)-\varepsilon \omega^{2} \rho_{\mathrm{f}} \mathbf{u}_{1, \varepsilon} \cdot \mathbf{n} \mathbf{n}=-\varepsilon^{2} \rho_{\mathrm{f}} \omega^{2} \mathbf{u}_{1} \cdot \mathbf{n} \mathbf{n} \quad \text { on } \quad \Gamma .
$$

Neglecting the term of order $\varepsilon^{2}$ in the previous right-hand side, we infer the condition (3.2).

Order 3

According to (5.6), (5.7), and (5.9), the truncated expansion $\mathbf{u}_{2, \varepsilon}=\mathbf{u}_{0}+\varepsilon \mathbf{u}_{1}+\varepsilon^{2} \mathbf{u}_{2}$ solves the elastic equation in $\Omega_{\mathrm{s}}$ with the condition

$$
\mathbf{T}\left(\mathbf{u}_{2, \varepsilon}\right)-\varepsilon \omega^{2} \rho_{\mathrm{f}}(1-\varepsilon \mathcal{H}) \mathbf{u}_{2, \varepsilon} \cdot \mathbf{n} \mathbf{n}=-\varepsilon^{3} \omega^{2} \rho_{\mathrm{f}} \mathbf{u}_{2} \cdot \mathbf{n} \mathbf{n}+\varepsilon^{3} \mathcal{H} \omega^{2} \rho_{\mathrm{f}}\left(\mathbf{u}_{1}+\varepsilon \mathbf{u}_{2}\right) \cdot \mathbf{n} \mathbf{n} .
$$

Neglecting the term of order $\varepsilon^{3}$ in the right-hand side, we obtain the condition (3.3). 
$\operatorname{Order} 4$

According to (5.6), (5.7), (5.9) and (5.11), the truncated expansion $\mathbf{u}_{3, \varepsilon}=\mathbf{u}_{0}+\varepsilon \mathbf{u}_{1}+\varepsilon^{2} \mathbf{u}_{2}+\varepsilon^{3} \mathbf{u}_{3}$ solves the elastic equation in $\Omega_{\mathrm{s}}$ with the condition

$$
\begin{aligned}
\mathbf{T}\left(\mathbf{u}_{3, \varepsilon}\right)-\varepsilon \omega^{2} \rho_{\mathrm{f}}[1-\varepsilon \mathcal{H} & \left.+\frac{\varepsilon^{2}}{3}\left(4 \mathcal{H}^{2}-\mathcal{K}+\Delta_{\Gamma}+\kappa^{2} \mathbb{I}\right)\right]\left(\mathbf{u}_{3, \varepsilon} \cdot \mathbf{n}\right) \mathbf{n}= \\
& -\varepsilon^{4} \rho_{\mathrm{f}} \omega^{2}\left[\left(\mathbf{u}_{3}-\mathcal{H}\left(\mathbf{u}_{2}+\varepsilon \mathbf{u}_{3}\right)\right) \cdot \mathbf{n}+\frac{1}{3}\left(4 \mathcal{H}^{2}-\mathcal{K}+\Delta_{\Gamma}+\kappa^{2} \mathbb{I}\right)\left(\left(\mathbf{u}_{1}+\varepsilon \mathbf{u}_{2}+\varepsilon^{2} \mathbf{u}_{3}\right) \cdot \mathbf{n}\right)\right] \mathbf{n} .
\end{aligned}
$$

Neglecting the previous right-hand side, we infer the condition (3.4).

\subsection{Estimates for the remainders}

The validation of the asymptotic expansion (5.1)-(5.2) consists in proving estimates for remainders $\left(\mathbf{r}_{\varepsilon}^{N}, r_{\varepsilon}^{N}\right)$ defined in $\Omega_{\mathrm{s}}$ and $\Omega_{\mathrm{f}}^{\varepsilon}$ as

$$
\mathbf{r}_{\varepsilon}^{N}=\mathbf{u}_{\varepsilon}-\sum_{n=0}^{N} \varepsilon^{n} \mathbf{u}_{n} \quad \text { in } \quad \Omega_{\mathbf{s}}, \quad \text { and } \quad r_{\varepsilon}^{N}(\mathbf{x})=\mathbf{p}_{\varepsilon}(\mathbf{x})-\sum_{n=0}^{N} \varepsilon^{n} \mathfrak{p}_{n}\left(y_{\alpha}, \frac{y_{3}}{\varepsilon}\right) \quad \text { for all } \mathbf{x} \in \Omega_{\mathbf{f}}^{\varepsilon} .
$$

The convergence result is the following statement.

Theorem 5.2. Under Assumptions 2.2, 2.4, 2.5, 2.6 and for $\varepsilon$ small enough, the solution $\left(\mathbf{u}_{\varepsilon}, \mathbf{p}_{\varepsilon}\right)$ of problem (2.1) has a two-scale expansion which can be written in the form (5.1), (5.2), with $\mathbf{u}_{j} \in \mathbf{H}^{1}\left(\Omega_{\mathbf{s}}\right)$ and $\mathfrak{p}_{j} \in \mathrm{H}^{1}(\Gamma \times(0,1))$. For each $N \in \mathbb{N}$, the remainders $\left(\mathbf{r}_{\varepsilon}^{N}, r_{\varepsilon}^{N}\right)$ satisfy

$$
\left\|\mathbf{r}_{\varepsilon}^{N}\right\|_{1, \Omega_{\mathrm{s}}}+\sqrt{\varepsilon}\left\|r_{\varepsilon}^{N}\right\|_{1, \Omega_{\mathrm{f}}^{\varepsilon}} \leqslant C_{N} \varepsilon^{N+1}
$$

with a constant $C_{N}$ independent of $\varepsilon$.

The error estimate (5.13) is obtained through an evaluation of the right-hand sides when applying Theorem 2.7 to the couple $(\mathbf{u}, \mathrm{p})=\left(\mathbf{r}_{\varepsilon}^{N}, r_{\varepsilon}^{N}\right)$.

Proof. The proof is rather standard, see for instance the proof of [7], Theorem 2.1 where the authors consider an interface problem for the Laplacian operator set in a domain with a thin layer. The error estimate (5.13) is obtained through an evaluation of the right-hand sides when the elasto-acoustic operator is applied to $\left(\mathbf{r}_{\varepsilon}^{N}, r_{\varepsilon}^{N}\right)$. By construction, the remainder $\left(\mathbf{r}_{\varepsilon}^{N}, r_{\varepsilon}^{N}\right)$ is solution of problem

$$
\begin{cases}\Delta r_{\varepsilon}^{N}+\kappa^{2} r_{\varepsilon}^{N}=f_{N, \varepsilon} & \text { in } \Omega_{\mathrm{f}}^{\varepsilon} \\ \nabla \cdot \underline{\underline{\sigma}}\left(\mathbf{r}_{\varepsilon}^{N}\right)+\omega^{2} \rho \mathbf{r}_{\varepsilon}^{N}=0 & \text { in } \Omega_{\mathrm{s}} \\ \partial_{\mathbf{n}} r_{\varepsilon}^{N}=\rho_{\mathrm{f}} \omega^{2} \mathbf{r}_{\varepsilon}^{N} \cdot \mathbf{n}+g_{N, \varepsilon} & \text { on } \Gamma \\ \mathbf{T}\left(\mathbf{r}_{\varepsilon}^{N}\right)=-r_{\varepsilon}^{N} \mathbf{n} & \text { on } \Gamma \\ r_{\varepsilon}^{N}=0 & \text { on } \Gamma^{\varepsilon} .\end{cases}
$$

Here, the right-hand sides are explicit:

$$
f_{N, \varepsilon}=\varepsilon^{N-1}\left[F_{N}-\sum_{l=1}^{N} \varepsilon^{l-2+N} R_{\varepsilon}^{N-l+1} \mathfrak{p}_{l}\right] \quad \text { in } \quad \Omega_{\mathrm{f}}^{\varepsilon},
$$

and

$$
g_{N, \varepsilon}=\rho_{\mathrm{f}} \omega^{2} \varepsilon^{N} \mathbf{u}^{N} \cdot \mathbf{n} \quad \text { on } \quad \Gamma .
$$


We have the following estimates for the residues $f_{N, \varepsilon}$ and $g_{N, \varepsilon}$

$$
\left\|f_{N, \varepsilon}\right\|_{0, \Omega_{\mathrm{f}}^{\varepsilon}}=\mathcal{O}\left(\varepsilon^{N-\frac{1}{2}}\right) \quad \text { and } \quad\left\|g_{N, \varepsilon}\right\|_{0, \Gamma}=\mathcal{O}\left(\varepsilon^{N}\right) .
$$

We can apply Theorem 2.7 to the couple $(\mathbf{u}, \mathrm{p})=\left(\mathbf{r}_{\varepsilon}^{N}, r_{\varepsilon}^{N}\right)$, and we obtain

$$
\left\|\mathbf{r}_{\varepsilon}^{N}\right\|_{1, \Omega_{\mathrm{s}}}+\left\|r_{\varepsilon}^{N}\right\|_{1, \Omega_{\mathrm{f}}^{\varepsilon}} \leqslant C_{N} \varepsilon^{N-\frac{1}{2}} .
$$

Writting $\mathbf{r}_{\varepsilon}^{N}=\mathbf{r}_{\varepsilon}^{N+2}+\varepsilon^{N+2} \mathbf{u}_{N+2}+\varepsilon^{N+1} \mathbf{u}_{N+1}$ and $r_{\varepsilon}^{N}=r_{\varepsilon}^{N+2}+\varepsilon^{N+2} \mathfrak{p}_{N+2}+\varepsilon^{N+1} \mathfrak{p}_{N+1}$, we apply the previous estimate to the couple $\left(\mathbf{r}_{\varepsilon}^{N+2}, r_{\varepsilon}^{N+2}\right)$ and we use estimates

$$
\left\|\mathbf{u}_{l}\right\|_{1, \Omega_{\mathrm{s}}}=\mathcal{O}(1) \quad \text { and } \quad\left\|\mathfrak{p}_{l}\right\|_{1, \Omega_{f}^{\varepsilon}}=\mathcal{O}\left(\varepsilon^{-\frac{1}{2}}\right)
$$

to infer the optimal estimates (5.13).

\subsection{Validation of equivalent conditions}

We consider the problem (3.1) with an equivalent condition and at a fixed frequency $\omega$ satisfying Assumption 2.4. The main result of this section is the following statement, that is for all $k \in\{0,1,2,3\}$ the problem (3.1) is well-posed in the space $\mathbf{V}^{k}$ (Not. 3.3), and its solution satisfies uniform $\mathbf{H}^{1}$ estimates.

Theorem 5.3. Under Assumptions 2.2, 2.4, 2.5, for all $k \in\{0,1,2,3\}$ there are constants $\varepsilon_{k}, C_{k}>0$ such that for all $\varepsilon \in\left(0, \varepsilon_{k}\right)$, the problem (3.1) with a data $\mathbf{f} \in \mathbf{L}^{2}\left(\Omega_{\mathbf{s}}\right)$ has a unique solution $\mathbf{u}_{\varepsilon}^{k} \in \mathbf{V}^{k}$ which satisfies the uniform estimates:

$$
\begin{gathered}
\left\|\mathbf{u}_{\varepsilon}^{k}\right\|_{1, \Omega_{\mathrm{s}}} \leqslant C_{k}\|\mathbf{f}\|_{0, \Omega_{\mathrm{s}}} \text { for all } k \in\{0,1,2\}, \\
\left\|\mathbf{u}_{\varepsilon}^{3}\right\|_{1, \Omega_{\mathrm{s}}}+\varepsilon^{\frac{3}{2}}\left\|\nabla_{\Gamma}\left(\mathbf{u}_{\varepsilon}^{3} \cdot \mathbf{n}\right)\right\|_{0, \Gamma} \leqslant C_{3}\|\mathbf{f}\|_{0, \Omega_{\mathbf{s}}} .
\end{gathered}
$$

The key for the proof of Theorem 5.3 is the following Lemma.

Lemma 5.4. Under Assumptions 2.2, 2.4, 2.5, for all $k \in\{0,1,2,3\}$ there exists constants $\varepsilon_{k}, C_{k}>0$ such that for all $\varepsilon \in\left(0, \varepsilon_{k}\right)$, any solution $\mathbf{u}_{\varepsilon}^{k} \in \mathbf{V}^{k}$ of problem (3.1) with a data $\mathbf{f} \in \mathbf{L}^{2}\left(\Omega_{\mathbf{s}}\right)$ satisfies the uniform estimate:

$$
\left\|\mathbf{u}_{\varepsilon}^{k}\right\|_{0, \Omega_{\mathrm{s}}} \leqslant C_{k}\|\mathbf{f}\|_{0, \Omega_{\mathrm{s}}}
$$

Remark 5.5. For $k=0$, the Theorem 5.3 and the Lemma 5.4 hold for all $\varepsilon>0$.

The Lemma 5.4 is proved in Section 6.1. As a consequence of this Lemma, each solution of the problem (3.1) satisfies uniform $\mathbf{H}^{1}$-estimates $(5.15 \mathrm{a}),(5.15 \mathrm{~b})$. Then, the proof of the Theorem 5.3 is obtained as a consequence of the Fredholm alternative since the problem (3.1) is of Fredholm type. One passes from Lemma 5.4 to Theorem 5.3 as from Lemma 4.2 to Theorem 4.1.

\section{Analysis of EQUivalent CONDitions}

In this section, we first prove the Lemma 5.4, i.e. uniform $\mathbf{L}^{2}$-estimate (5.16) for the solution of problem (3.1), Section 6.1. In Section 6.2, we prove that the solution $\mathbf{u}_{\varepsilon}^{k}$ of problem (3.1) satisfies uniform $\mathbf{H}^{1}$ error estimates (3.5) and we infer the Theorem 3.4. We focus on the proof of Lemma 5.4 for $k=3$ only since the proof when $k \in\{0,1,2\}$ is simpler. Hence we consider the problem (here $\mathbf{u}=\mathbf{u}_{\varepsilon}^{3}$ )

$$
\begin{cases}\nabla \cdot \underline{\underline{\sigma}}(\mathbf{u})+\omega^{2} \rho \mathbf{u}=\mathbf{f} & \text { in } \quad \Omega_{\mathrm{s}} \\ \mathbf{T}(\mathbf{u})+\mathrm{B}_{3, \varepsilon}(\mathbf{u} \cdot \mathbf{n}) \mathbf{n}=0 & \text { on } \quad \Gamma .\end{cases}
$$


To prepare for the proof, we introduce the variational formulation for $\mathbf{u}_{\varepsilon}$. If $\mathbf{u} \in \mathbf{V}^{3}$ is a solution of (6.1), then it satisfies for all $\mathbf{v} \in \mathbf{V}^{3}$ :

$$
\int_{\Omega_{\mathrm{s}}}\left(\underline{\underline{\sigma}}(\mathbf{u}): \underline{\underline{\epsilon}}(\overline{\mathbf{v}})-\omega^{2} \rho \mathbf{u} \overline{\mathbf{v}}\right) \mathrm{d} \mathbf{x}-\varepsilon \omega^{2} \rho_{\mathrm{f}} \int_{\Gamma} \mathcal{J}_{\varepsilon} \mathbf{u} \cdot \mathbf{n} \overline{\mathbf{v}} \cdot \mathbf{n} \mathrm{d} \sigma+\frac{\varepsilon^{3}}{3} \omega^{2} \rho_{\mathrm{f}} \int_{\Gamma} \nabla_{\Gamma}(\mathbf{u} \cdot \mathbf{n}) \nabla_{\Gamma}(\overline{\mathbf{v}} \cdot \mathbf{n}) \mathrm{d} \sigma=-\int_{\Omega_{\mathrm{s}}} \mathbf{f} \cdot \overline{\mathbf{v}} \mathrm{d} \mathbf{x}
$$

where $\mathcal{J}_{\varepsilon}$ is a function defined on $\Gamma$ as $\mathcal{J}_{\varepsilon}=\left(1-\varepsilon \mathcal{H}+\frac{\varepsilon^{2}}{3}\left(4 \mathcal{H}^{2}-\mathcal{K}-\kappa^{2}\right)\right)$ which tends to 1 when $\varepsilon$ goes to 0 .

\subsection{Proof of Lemma 5.4: Uniform $\mathrm{L}^{2}$-estimate of the elastic displacement}

Reductio ad absurdum: we assume that there is a sequence $\left(\mathbf{u}_{m}\right) \in \mathbf{L}^{2}\left(\Omega_{\mathbf{s}}\right), m \in \mathbb{N}$, of solutions of the problem (6.1) associated with a parameter $\varepsilon_{m}$ and a right-hand side $\mathbf{f}_{m} \in \mathbf{L}^{2}\left(\Omega_{\mathbf{s}}\right)$ :

$$
\begin{gathered}
\nabla \cdot \underline{\underline{\sigma}}\left(\mathbf{u}_{m}\right)+\omega^{2} \rho \mathbf{u}_{m}=\mathbf{f}_{m} \quad \text { in } \quad \Omega_{\mathbf{s}} \\
\mathbf{T}\left(\mathbf{u}_{m}\right)-\varepsilon_{m} \omega^{2} \rho_{\mathrm{f}} \mathcal{J}_{\varepsilon_{m}} \mathbf{u}_{m} \cdot \mathbf{n} \mathbf{n}-\frac{\varepsilon_{m}^{3}}{3} \omega^{2} \rho_{\mathrm{f}} \Delta_{\Gamma}\left(\mathbf{u}_{m} \cdot \mathbf{n}\right) \mathbf{n}=0 \quad \text { on } \quad \Gamma
\end{gathered}
$$

satisfying the following conditions

$$
\begin{array}{cl}
\varepsilon_{m} \rightarrow 0 & \text { as } m \rightarrow \infty \\
\left\|\mathbf{u}_{m}\right\|_{0, \Omega_{\mathrm{s}}}=1 & \text { for all } m \in \mathbb{N}, \\
\left\|\mathbf{f}_{m}\right\|_{0, \Omega_{\mathrm{s}}} \rightarrow 0 & \text { as } m \rightarrow \infty .
\end{array}
$$

\subsubsection{Estimates of the sequence $\left\{\mathbf{u}_{m}\right\}$}

We first prove that the sequence $\left\{\mathbf{u}_{m}\right\}$ is bounded (only) in $\mathbf{H}^{1}\left(\Omega_{\mathbf{s}}\right)$. We particularize the variational formulation (6.2) for the sequence $\left\{\mathbf{u}_{m}\right\}$ : for all $\mathbf{v} \in \mathbf{V}^{3}$,

$$
\begin{aligned}
\int_{\Omega_{\mathrm{s}}}\left(\underline{\underline{\sigma}}\left(\mathbf{u}_{m}\right): \underline{\underline{\epsilon}}(\overline{\mathbf{v}})-\omega^{2} \rho \mathbf{u}_{m} \overline{\mathbf{v}}\right) \mathrm{d} \mathbf{x}-\varepsilon_{m} \omega^{2} \rho_{\mathrm{f}} \int_{\Gamma} \mathcal{J}_{\varepsilon_{m}} \mathbf{u}_{m} \cdot \mathbf{n} \overline{\mathbf{v}} \cdot \mathbf{n} \mathrm{d} \sigma \\
\quad+\frac{\varepsilon_{m}^{3}}{3} \omega^{2} \rho_{\mathrm{f}} \int_{\Gamma} \nabla_{\Gamma}\left(\mathbf{u}_{m} \cdot \mathbf{n}\right) \nabla_{\Gamma}(\overline{\mathbf{v}} \cdot \mathbf{n}) \mathrm{d} \sigma=-\int_{\Omega_{\mathrm{s}}} \mathbf{f}_{m} \cdot \overline{\mathbf{v}} \mathrm{d} \mathbf{x} .
\end{aligned}
$$

Choosing $\mathbf{v}=\mathbf{u}_{m}$ in (6.5), we obtain with the help of condition (6.4b) the uniform bound

$$
\int_{\Omega_{\mathrm{s}}} \underline{\underline{C}} \underline{\underline{\epsilon}}\left(\mathbf{u}_{m}\right): \underline{\underline{\epsilon}}\left(\overline{\mathbf{u}}_{m}\right) \mathrm{d} \mathbf{x}-\varepsilon_{m} \omega^{2} \rho_{\mathrm{f}} \int_{\Gamma} \mathcal{J}_{\varepsilon_{m}}\left|\mathbf{u}_{m} \cdot \mathbf{n}\right|^{2} \mathrm{~d} \sigma+\frac{\varepsilon_{m}^{3}}{3} \omega^{2} \rho_{\mathrm{f}} \int_{\Gamma}\left|\nabla_{\Gamma}\left(\mathbf{u}_{m} \cdot \mathbf{n}\right)\right|^{2} \mathrm{~d} \sigma \leqslant \omega^{2} \rho+\left\|\mathbf{f}_{m}\right\|_{0, \Omega_{\mathrm{s}}}
$$

Since the tensor $\underline{\underline{\epsilon}}(\mathbf{u})$ is symmetric, thanks to Assumptions 2.2 (i)-(iii) together with the Korn inequality, we infer: there exists constants $C, c>0$ such that for all $\mathbf{u} \in \mathbf{H}^{1}\left(\Omega_{\mathrm{s}}\right)$

$$
\int_{\Omega_{\mathrm{s}}} \underline{\underline{C}} \underline{\underline{\epsilon}}(\mathbf{u}): \underline{\underline{\epsilon}}(\overline{\mathbf{u}}) \mathrm{d} \mathbf{x} \geqslant \alpha C\|\mathbf{u}\|_{1, \Omega_{\mathrm{s}}}^{2}-\alpha c\|\mathbf{u}\|_{0, \Omega_{\mathrm{s}}}^{2}
$$

Combining the previous inequality (6.7) and the trace inequality

$$
\forall \mathbf{u} \in \mathbf{H}^{1}\left(\Omega_{\mathrm{s}}\right), \quad\|\mathbf{u} \cdot \mathbf{n}\|_{0, \Gamma} \leqslant C_{1}\|\mathbf{u}\|_{1, \Omega_{\mathrm{s}}}
$$

we infer for $m$ large enough

$$
\alpha C\left\|\mathbf{u}_{m}\right\|_{1, \Omega_{\mathrm{s}}}^{2}-\varepsilon_{m} \beta \omega^{2} \rho_{\mathrm{f}}\left\|\mathbf{u}_{m} \cdot \mathbf{n}\right\|_{0, \Gamma}^{2}+\frac{\varepsilon_{m}^{3}}{3} \omega^{2} \rho_{\mathrm{f}}\left\|\nabla_{\Gamma}\left(\mathbf{u}_{m} \cdot \mathbf{n}\right)\right\|_{0, \Gamma}^{2} \leqslant C_{2}+\left\|\mathbf{f}_{m}\right\|_{0, \Omega_{\mathrm{s}}}
$$


with a constant $\beta>0$ such that $\mathcal{J}_{\varepsilon} \leqslant \beta$ for $\varepsilon$ small enough (remind $\mathcal{J}_{\varepsilon} \rightarrow 1$ as $\varepsilon \rightarrow 0$ ), and $C_{2}=\alpha c+\omega^{2} \rho$. Using again the trace inequality (6.8), we obtain

$$
\left(\alpha C-\varepsilon_{m} \beta \omega^{2} \rho_{\mathrm{f}} C_{1}^{2}\right)\left\|\mathbf{u}_{m}\right\|_{1, \Omega_{\mathrm{s}}}^{2}+\frac{\varepsilon_{m}^{3}}{3} \omega^{2} \rho_{\mathrm{f}}\left\|\nabla_{\Gamma}\left(\mathbf{u}_{m} \cdot \mathbf{n}\right)\right\|_{0, \Gamma}^{2} \leqslant C_{2}+\left\|\mathbf{f}_{m}\right\|_{0, \Omega_{\mathrm{s}}} .
$$

Then, using (6.4a), there holds: $\alpha C-\varepsilon_{m} \beta \omega^{2} \rho_{\mathrm{f}} C_{1}^{2}>0$, for $m$ large enough. According to (6.4c) we infer that the sequence $\left\{\mathbf{u}_{m}\right\}$, resp. $\left\{\left(\varepsilon_{m}\right)^{\frac{3}{2}} \nabla_{\Gamma}\left(\mathbf{u}_{m} \cdot \mathbf{n}\right)\right\}$, is bounded in $\mathbf{H}^{1}\left(\Omega_{\mathrm{s}}\right)$, resp. in $\mathbf{L}^{2}(\Gamma)$ :

$$
\begin{gathered}
\left\|\mathbf{u}_{m}\right\|_{1, \Omega_{\mathrm{s}}} \leqslant C, \\
\left(\varepsilon_{m}\right)^{\frac{3}{2}}\left\|\nabla_{\Gamma}\left(\mathbf{u}_{m} \cdot \mathbf{n}\right)\right\|_{0, \Gamma} \leqslant C .
\end{gathered}
$$

Another consequence of $(6.9 \mathrm{a})$ is that the sequence $\left\{\mathbf{u}_{m} \cdot \mathbf{n}\right\}$ is bounded in $\mathrm{H}^{\frac{1}{2}}(\Gamma)$.

$$
\left\|\mathbf{u}_{m} \cdot \mathbf{n}\right\|_{\frac{1}{2}, \Gamma} \leqslant C .
$$

\subsubsection{Limit of the sequence and conclusion}

The domain $\Omega_{\mathbf{s}}$ being bounded, the embedding of $\mathbf{H}^{1}\left(\Omega_{\mathbf{s}}\right)$ in $\mathbf{L}^{2}\left(\Omega_{\mathbf{s}}\right)$ is compact. Hence as a consequence of (6.9a), using the Rellich Lemma we can extract a subsequence of $\left\{\mathbf{u}_{m}\right\}$ (still denoted by $\left\{\mathbf{u}_{m}\right\}$ ) which is converging in $\mathbf{L}^{2}\left(\Omega_{\mathbf{s}}\right)$, and we can assume that the sequence $\left\{\underline{\underline{\nabla}} \mathbf{u}_{m}\right\}$ is strongly converging in $\mathbf{L}^{2}\left(\Omega_{\mathbf{s}}\right)$. As a consequence of (6.10), up to the extraction of a subsequence, we can assume that the sequence $\left\{\mathbf{u}_{m} \cdot \mathbf{n}\right\}$ is strongly converging in $\mathrm{L}^{2}(\Gamma)$ : we deduce that there is $\mathbf{u} \in \mathbf{L}^{2}\left(\Omega_{\mathbf{s}}\right)$ such that

$$
\left\{\begin{array}{lll}
\underline{\epsilon}\left(\mathbf{u}_{m}\right) \rightarrow \underline{\epsilon}(\mathbf{u}) & \text { in } & \mathbf{L}^{2}\left(\Omega_{\mathrm{s}}\right) \\
\mathbf{u}_{m} \rightarrow \mathbf{u} & \text { in } & \mathbf{L}^{2}\left(\Omega_{\mathrm{s}}\right) \\
\mathbf{u}_{m} \cdot \mathbf{n} \rightarrow \mathbf{u} \cdot \mathbf{n} & \text { in } & \mathrm{L}^{2}(\Gamma) .
\end{array}\right.
$$

A consequence of the strong convergence in $\mathbf{L}^{2}\left(\Omega_{\mathbf{s}}\right)$ and $(6.4 \mathrm{~b})$ is that $\|\mathbf{u}\|_{0, \Omega_{\mathbf{s}}}=1$. As a consequence of $(6.9 \mathrm{~b})$, we can extract a subsequence of $\left\{\left(\varepsilon_{m}\right)^{\frac{3}{2}} \nabla_{\Gamma}\left(\mathbf{u}_{m} \cdot \mathbf{n}\right)\right\}$ (still denoted by $\left\{\left(\varepsilon_{m}\right)^{\frac{3}{2}} \nabla_{\Gamma}\left(\mathbf{u}_{m} \cdot \mathbf{n}\right)\right\}$ ) which is weakly converging to a function $\mathbf{t} \in \mathbf{L}^{2}(\Gamma)$

$$
\left(\varepsilon_{m}\right)^{\frac{3}{2}} \nabla_{\Gamma}\left(\mathbf{u}_{m} \cdot \mathbf{n}\right) \rightarrow \mathbf{t} \quad \text { in } \quad \mathbf{L}^{2}(\Gamma)
$$

Using Assumption 2.4, we are going to prove that $\mathbf{u}=0$, which will contradict $\|\mathbf{u}\|_{0, \Omega_{\mathrm{s}}}=1$, and finally prove estimate (5.16). Let $\mathbf{v} \in \mathbf{V}^{3}$ be a test function in (6.5)

$$
\begin{aligned}
\int_{\Omega_{\mathrm{s}}}\left(\underline{\underline{\sigma}}\left(\mathbf{u}_{m}\right): \underline{\underline{\epsilon}}(\overline{\mathbf{v}})-\omega^{2} \rho \mathbf{u}_{m} \overline{\mathbf{v}}\right) \mathrm{d} \mathbf{x}-\varepsilon_{m} \omega^{2} \rho_{\mathrm{f}} \int_{\Gamma} \mathcal{J}_{\varepsilon_{m}} \mathbf{u}_{m} \cdot \mathbf{n} \overline{\mathbf{v}} \cdot \mathbf{n} \mathrm{d} \sigma \\
\quad+\frac{\varepsilon_{m}^{3}}{3} \omega^{2} \rho_{\mathrm{f}} \int_{\Gamma} \nabla_{\Gamma}\left(\mathbf{u}_{m} \cdot \mathbf{n}\right) \nabla_{\Gamma}(\overline{\mathbf{v}} \cdot \mathbf{n}) \mathrm{d} \sigma=-\int_{\Omega_{\mathrm{s}}} \mathbf{f}_{m} \cdot \overline{\mathbf{v}} \mathrm{d} \mathbf{x} .
\end{aligned}
$$

According to (6.11), (6.12), and (6.4a), taking limits as $m \rightarrow+\infty$, there holds

$$
\varepsilon_{m} \int_{\Gamma} \mathcal{J}_{\varepsilon_{m}} \mathbf{u}_{m} \cdot \mathbf{n} \overline{\mathbf{v}} \cdot \mathbf{n} \mathrm{d} \sigma \rightarrow 0 \quad \text { and } \quad \frac{\varepsilon_{m}^{3}}{3} \int_{\Gamma} \nabla_{\Gamma}\left(\mathbf{u}_{m} \cdot \mathbf{n}\right) \nabla_{\Gamma}(\overline{\mathbf{v}} \cdot \mathbf{n}) \mathrm{d} \sigma \rightarrow 0 .
$$

Hence, according to (6.11), (6.4a) and (6.4c), taking limits as $m \rightarrow+\infty$, we deduce from the previous equalities $(6.13) \mathbf{u} \in \mathbf{H}^{1}\left(\Omega_{\mathrm{s}}\right)$ satisfies for all $\mathbf{v} \in \mathbf{H}^{1}\left(\Omega_{\mathrm{s}}\right)$ :

$$
\int_{\Omega_{\mathrm{s}}}\left(\underline{\underline{\sigma}}(\mathbf{u}): \underline{\underline{\epsilon}}(\overline{\mathbf{v}})-\omega^{2} \rho \mathbf{u} \overline{\mathbf{v}}\right) \mathrm{d} \mathbf{x}=0
$$


Integrating by parts we find that $\mathbf{u}$ satisfies the problem

$$
\left\{\begin{array}{lll}
\nabla \cdot \underline{\sigma}(\mathbf{u})+\omega^{2} \rho \mathbf{u}=0 & \text { in } & \Omega_{\mathbf{s}} \\
\mathbf{T}(\mathbf{u})=0 & \text { on } \Gamma
\end{array}\right.
$$

By Assumption 2.4, we deduce

$$
\mathbf{u}=0 \quad \text { in } \quad \Omega_{\mathbf{s}}
$$

which contradicts $\|\mathbf{u}\|_{0, \Omega_{\mathrm{s}}}=1$ and ends the proof of Lemma 5.4.

\subsection{Proof of error estimates}

In this section we prove Theorem 3.4. Since the problem (3.1) is of Fredholm type, it is sufficient to show that any solution $\mathbf{u}_{\varepsilon}^{k}$ of (3.1) satisfies the error estimate (3.5)

$$
\left\|\mathbf{u}_{\varepsilon}-\mathbf{u}_{\varepsilon}^{k}\right\|_{1, \Omega_{\mathrm{s}}} \leqslant C \varepsilon^{k+1}
$$

We prove hereafter the estimate (3.5) in two steps, Sections 6.2.1 and 6.2.2.

\subsubsection{Step A}

The first step consists to derive an expansion of $\mathbf{u}_{\varepsilon}^{k}$ and to show that the truncated expansions of $\mathbf{u}_{\varepsilon}^{k}$ and $\mathbf{u}_{\varepsilon}$ coincide up to the order $\varepsilon^{k}$ :

$$
\begin{aligned}
& \mathbf{u}_{\varepsilon}=\mathbf{u}_{0}+\varepsilon \mathbf{u}_{1}+\varepsilon^{2} \mathbf{u}_{2}+\ldots+\varepsilon^{k} \mathbf{u}_{k}+\mathbf{r}_{\varepsilon}^{k}, \\
& \mathbf{u}_{\varepsilon}^{k}=\mathbf{u}_{0}+\varepsilon \mathbf{u}_{1}+\varepsilon^{2} \mathbf{u}_{2}+\ldots+\varepsilon^{k} \mathbf{u}_{k}+\tilde{\mathbf{r}}_{\varepsilon}^{k} .
\end{aligned}
$$

Hereafter, we justify the expansion (6.15). By construction, $\mathbf{u}_{\varepsilon}^{k}$ admits an expansion

$$
\mathbf{u}_{\varepsilon}^{k}=\mathbf{v}_{0}+\varepsilon \mathbf{v}_{1}+\varepsilon^{2} \mathbf{v}_{2}+\ldots+\varepsilon^{k} \mathbf{v}_{k}+\tilde{\mathbf{r}}_{\varepsilon}^{k}
$$

where each term $\mathbf{v}_{n}$, for $0 \leqslant n \leqslant k$, satisfies the problem (5.5) as well as the term $\mathbf{u}_{n}$. Using the spectral Assumption 2.4, we infer that for all $0 \leqslant n \leqslant k, \mathbf{v}_{n}=\mathbf{u}_{n}$ in $\Omega_{\mathbf{s}}$, and the expansion (6.15) holds.

Hence,

$$
\left\|\mathbf{u}_{\varepsilon}-\mathbf{u}_{\varepsilon}^{k}\right\|_{1, \Omega_{\mathrm{s}}}=\left\|\mathbf{r}_{\varepsilon}^{k}-\tilde{\mathbf{r}}_{\varepsilon}^{k}\right\|_{1, \Omega_{\mathrm{s}}} .
$$

The estimate of the remainder $\mathbf{r}_{\varepsilon}^{k}$ is already proved in Theorem 5.2 (Sect. 5.4): $\left\|\mathbf{r}_{\varepsilon}^{k}\right\|_{1, \Omega_{\mathrm{s}}} \leqslant C \varepsilon^{k+1}$. In the next step, we prove estimates for the remainder $\tilde{\mathbf{r}}_{\varepsilon}^{k}$.

\subsubsection{Step $B$}

According to (6.15), the remainder $\tilde{\mathbf{r}}_{\varepsilon}^{k}$ satisfies the elastic equation in $\Omega_{\mathbf{s}}$. We apply the operator $\mathbf{T}+\mathbf{B}_{k, \varepsilon}$ (where $\mathbf{B}_{k, \varepsilon}(\mathbf{u}):=\mathrm{B}_{k, \varepsilon}(\mathbf{u} \cdot \mathbf{n}) \mathbf{n}$ ) to the remainder $\tilde{\mathbf{r}}_{\varepsilon}^{k}$. Then, we prove hereafter that

$$
\mathbf{T}\left(\tilde{\mathbf{r}}_{\varepsilon}^{k}\right)+\mathrm{B}_{k, \varepsilon}\left(\tilde{\mathbf{r}}_{\varepsilon}^{k} \cdot \mathbf{n}\right) \mathbf{n}=\mathcal{O}\left(\varepsilon^{k+1}\right) \quad \text { on } \quad \Gamma .
$$

Since $\mathbf{u}_{\varepsilon}^{0}=\mathbf{u}_{0}$, then $\tilde{\mathbf{r}}_{\varepsilon}^{0}=0$. Relying on the construction of equivalent conditions detailed in Section 5.3, there holds

$$
\begin{aligned}
& \mathbf{T}\left(\tilde{\mathbf{r}}_{\varepsilon}^{1}\right)+\mathrm{B}_{1, \varepsilon}\left(\tilde{\mathbf{r}}_{\varepsilon}^{1} \cdot \mathbf{n}\right) \mathbf{n}=\varepsilon^{2} \omega^{2} \rho_{\mathrm{f}} \mathbf{u}_{1} \cdot \mathbf{n} \mathbf{n} \\
& \mathbf{T}\left(\tilde{\mathbf{r}}_{\varepsilon}^{2}\right)+\mathrm{B}_{2, \varepsilon}\left(\tilde{\mathbf{r}}_{\varepsilon}^{2} \cdot \mathbf{n}\right) \mathbf{n}=\varepsilon^{3} \omega^{2} \rho_{\mathrm{f}}\left((1-\varepsilon \mathcal{H}) \mathbf{u}_{2}+\mathcal{H} \mathbf{u}_{1}\right) \cdot \mathbf{n} \mathbf{n} \\
& \mathbf{T}\left(\tilde{\mathbf{r}}_{\varepsilon}^{3}\right)+\mathrm{B}_{3, \varepsilon}\left(\tilde{\mathbf{r}}_{\varepsilon}^{3} \cdot \mathbf{n}\right) \mathbf{n}=\varepsilon^{4} \omega^{2} \rho_{\mathrm{f}}\left[\left(\mathbf{u}_{3}-\mathcal{H}\left(\mathbf{u}_{2}+\varepsilon \mathbf{u}_{3}\right)\right) \cdot \mathbf{n}+\frac{1}{3}\left(4 \mathcal{H}^{2}-\mathcal{K}+\Delta_{\Gamma}+\kappa^{2} \mathbb{I}\right)\left(\left(\mathbf{u}_{1}+\varepsilon \mathbf{u}_{2}+\varepsilon^{2} \mathbf{u}_{3}\right) \cdot \mathbf{n}\right)\right] \mathbf{n}
\end{aligned}
$$

on $\Gamma$. According to the estimate (5.16), we infer the uniform estimate

$$
\left\|\tilde{\mathbf{r}}_{\varepsilon}^{k}\right\|_{1, \Omega_{\mathrm{s}}} \leqslant C \varepsilon^{k+1}
$$

which ends the proof of Theorem 3.4. 
Acknowledgements. The author thanks very warmly Monique Dauge for her well-considered suggestions.

\section{REFERENCES}

[1] T. Abboud and H. Ammari, Diffraction at a curved grating: TM and TE cases, homogenization. J. Math. Anal. Appl. 202 (1996) 995-1026.

[2] H. Ammari, E. Beretta, E. Francini, H. Kang and M. Lim, Reconstruction of small interface changes of an inclusion from modal measurements ii: The elastic case. J. Math. Pures Appl. 94 (2010) 322-339.

[3] H. Ammari and J.C. Nédélec, Time-harmonic electromagnetic fields in thin chiral curved layers. SIAM J. Math. Anal. 29 (1998) 395-423.

[4] V. Andreev and A. Samarski, Méthode aux différences pour les équations elliptiques. Edition de Moscou, Moscou (1978).

[5] X. Antoine, H. Barucq and L. Vernhet, High-frequency asymptotic analysis of a dissipative transmission problem resulting in generalized impedance boundary conditions. Asymptot. Anal. 26 (2001) 257-283.

[6] A. Bendali and K. Lemrabet, The effect of a thin coating on the scattering of a time-harmonic wave for the Helmholtz equation. SIAM J. Appl. Math. 56 (1996) 1664-1693.

[7] G. Caloz, M. Costabel, M. Dauge and G. Vial, Asymptotic expansion of the solution of an interface problem in a polygonal domain with thin layer. Asymptot. Anal. 50 (2006) 121-173.

[8] G. Caloz, M. Dauge, E. Faou and V. Péron, On the influence of the geometry on skin effect in electromagnetism. Comput. Methods Appl. Mech. Engrg. 200 (2011) 1053-1068.

[9] M. Costabel, M. Dauge and S. Nicaise, Corner Singularities and Analytic Regularity for Linear Elliptic Systems. Part I: Smooth domains (2010) hal-00453934.

[10] J. Diaz and V. Péron, Equivalent Conditions for Elasto-Acoustics, in Waves 2013: The 11th International Conference on Math. Numer. Aspects of Waves. Gammarth, Tunisie (2013) 345-346.

[11] M. Durán and J.-C. Nédélec, Un problème spectral issu d'un couplage élasto-acoustique. ESAIM: M2AN 34 (2000) 835-857.

[12] B. Engquist and J.C. Nédélec, Effective boundary condition for acoustic and electromagnetic scattering in thin layers. Technical Report of CMAP 278 (1993).

[13] H. Haddar, P. Joly and H.-M. Nguyen, Generalized impedance boundary conditions for scattering problems from strongly absorbing obstacles: the case of Maxwell's equations. Math. Models Methods Appl. Sci. 18 (2008) 1787-1827.

[14] T. Huttunen, J.P. Kaipio and P. Monk, An ultra-weak method for acoustic fluid-solid interaction. J. Comput. Appl. Math. 213 (2008) 166-185.

[15] D.S. Jones, Low-frequency scattering by a body in lubricated contact. Quart. J. Mech. Appl. Math. 36 (1983) $111-138$.

[16] O.D. Lafitte, Diffraction in the high frequency regime by a thin layer of dielectric material. I. The equivalent impedance boundary condition. SIAM J. Appl. Math. 59 (1999) 1028-1052.

[17] O.D. Lafitte and G. Lebeau, Équations de Maxwell et opérateur d'impédance sur le bord d'un obstacle convexe absorbant. C. R. Acad. Sci. Paris Sér. I Math. 316 (1993) 1177-1182.

[18] K. Lemrabet, Le problème de Ventcel pour le système de l'élasticité dans un domaine de $\mathbf{R}^{3}$. C. R. Acad. Sci. Paris Sér. I Math. 304 (1987) 151-154.

[19] M.A. Leontovich, Approximate boundary conditions for the electromagnetic field on the surface of a good conductor, in Investigations on radiowave propagation, vol. 2. Printing House of the USSR Academy of Sciences, Moscow (1948) 5-12.

[20] C.J. Luke and P.A. Martin, Fluid-solid interaction: acoustic scattering by a smooth elastic obstacle. SIAM J. Appl. Math. 55 (1995) 904-922.

[21] P. Monk and V. Selgas, An inverse fluid-solid interaction problem. Inverse Probl. Imaging 3 (2009) $173-198$.

[22] D. Natroshvili, A.-M. Sändig and W.L. Wendland, Fluid-structure interaction problems, in Mathematical aspects of boundary element methods (Palaiseau, 1998), vol. 414. Research Notes Math. Chapman \& Hall/CRC, Boca Raton, FL (2000) $252-262$.

[23] V. Péron, Equivalent Boundary Conditions for an Elasto-Acoustic Problem set in a Domain with a Thin Layer. Rapport de recherche RR-8163, INRIA (2013).

[24] S.M. Rytov, Calcul du skin effect par la méthode des perturbations. J. Phys. 11 (1940) 233-242.

[25] T.B.A. Senior and J.L. Volakis, and Institution of Electrical Engineers. Approximate Boundary Conditions in Electromagnetics. IEE Electromagnetic Waves Series. Inst of Engineering \& Technology (1995). 